\title{
On the mean-field limit of bosons with Coulomb two-body interaction
}

\section{Journal Article}

\section{Author(s):}

Fröhlich, Jürg; Knowles, Antti; Schwarz, Simon

Publication date:

2009-06

Permanent link:

https://doi.org/10.3929/ethz-b-000016271

Rights / license:

In Copyright - Non-Commercial Use Permitted

\section{Originally published in:}

Communications in Mathematical Physics 288(3), https://doi.org/10.1007/s00220-009-0754-z 


\title{
On the Mean-Field Limit of Bosons with Coulomb Two-Body Interaction
}

\author{
Jürg Fröhlich, Antti Knowles, Simon Schwarz \\ Institute of Theoretical Physics, ETH Hönggerberg, CH-8093 Zürich, Switzerland. \\ E-mail: juerg@itp.phys.ethz.ch; aknowles@itp.phys.ethz.ch; sschwarz@itp.phys.ethz.ch
}

Received: 28 May 2008 / Accepted: 2 December 2008

Published online: 28 February 2009 - (C) Springer-Verlag 2009

\begin{abstract}
In the mean-field limit the dynamics of a quantum Bose gas is described by a Hartree equation. We present a simple method for proving the convergence of the microscopic quantum dynamics to the Hartree dynamics when the number of particles becomes large and the strength of the two-body potential tends to 0 like the inverse of the particle number. Our method is applicable for a class of singular interaction potentials including the Coulomb potential. We prove and state our main result for the Heisenbergpicture dynamics of "observables", thus avoiding the use of coherent states. Our formulation shows that the mean-field limit is a "semi-classical" limit.
\end{abstract}

\section{Introduction}

Whenever many particles interact by means of weak two-body potentials, one expects that the potential felt by any one particle is given by an average potential generated by the particle density. In this mean-field regime, one hopes to find that the emerging dynamics is simpler and less encumbered by tedious microscopic information than the original $N$-body dynamics.

The mathematical study of such problems has quite a long history. In the context of classical mechanics, where the mean-field limit is described by the Vlasov equation, the problem was successfully studied by Braun and Hepp [3], as well as Neunzert [16]. The mean-field limit of quantum Bose gases was first addressed in the seminal paper [10] of Hepp. We refer to [6] for a short discussion of some subsequent results. The case with a Coulomb interaction potential was treated by Erdős and Yau in [6]. Recently, Rodnianski and Schlein [21] have derived explicit estimates for the rate of convergence to the meanfield limit, using the methods of [10 and 9]. A sharper bound on the rate of convergence in the case of a sufficiently regular interaction potential was derived by Schlein and Erdős [22], by using a new method inspired by Lieb-Robinson inequalities. In [7,15], the mean-field limit $(N \rightarrow \infty)$ and the classical limit were studied simultaneously. A conceptually quite novel approach to studying mean-field limits was introduced in [8]. 
In that paper, the time evolution of quantum and corresponding "classical" observables is studied in the Heisenberg picture, and it is shown that "time evolution commutes with quantisation" up to terms that tend to 0 in the mean-field ("classical") limit, which is a Egorov-type result.

In this paper we present a new, simpler way of handling singular interaction potentials. It yields a Egorov-type formulation of convergence to the mean-field limit, thus obviating the need to consider particular (traditionally coherent) states as initial conditions. Another, technical, advantage of our method is that it requires no regularity (traditionally $H^{1}$ - or $H^{2}$-regularity) when applied to coherent states.

Such kinds of results were first obtained by Egorov [5] for the semi-classical limit of a quantum system. Roughly, the statement is that time-evolution commutes with quantisation in the semi-classical limit. We sketch this in a simple example: Let us start with a classical Hamiltonian system of a finite number $f$ of degrees of freedom. The classical algebra of observables $\mathfrak{A}$ is given by (some subalgebra of) the Abelian algebra of smooth functions on the phase space $\Gamma:=\mathbb{R}^{2 f}$. Let $H \in \mathfrak{A}$ be a Hamilton function. Together with the symplectic structure on $\Gamma, H$ generates a symplectic flow $\phi^{t}$ on $\Gamma$. Now we define a quantisation map $\widehat{(\cdot)_{\hbar}}: \mathfrak{A} \rightarrow \widehat{\mathfrak{A}}$, where $\widehat{\mathfrak{A}}$ is some subalgebra of $\mathcal{B}\left(L^{2}\left(\mathbb{R}^{f}\right)\right)$. For concreteness, let $\widehat{(\cdot)} \hbar$ be Weyl quantisation with deformation parameter $\hbar$. This implies that

$$
\left.\left[\widehat{A}_{\hbar}, \widehat{B}_{\hbar}\right]=\frac{\hbar}{\mathrm{i}} \widehat{\{A, B}\right\}_{\hbar}+O\left(\hbar^{2}\right)
$$

for $\hbar \rightarrow 0$. The quantised Hamilton function defines a 1-parameter group of automorphisms on $\widehat{\mathfrak{A}}$ through

$$
\mathbf{A} \mapsto \mathrm{e}^{\mathrm{i} t \widehat{H}_{\hbar} / \hbar} \mathbf{A} \mathrm{e}^{-\mathrm{i} t \widehat{H}_{\hbar} / \hbar}, \quad \mathbf{A} \in \widehat{\mathfrak{A}} .
$$

A Egorov-type semi-classical result states that, for all $A \in \mathfrak{A}$ and $t \in \mathbb{R}$,

$$
\left(\widehat{A \circ \phi^{t}}\right)_{\hbar}=\mathrm{e}^{\mathrm{i} t \widehat{H}_{\hbar} / \hbar} \widehat{A}_{\hbar} \mathrm{e}^{-\mathrm{i} t \widehat{H}_{\hbar} / \hbar}+R_{\hbar}(t),
$$

where $\left\|R_{\hbar}(t)\right\| \rightarrow 0$ as $\hbar \rightarrow 0$.

This approach identifies the semi-classical limit as the converse of quantisation. In a similar fashion, we identify the mean-field limit as the converse of "second quantisation". In this case the deformation parameter is not $\hbar$, but $N^{-1}$, a parameter proportional to the coupling constant. We consider the mean-field dynamics (given by the Hartree equation in the case of bosons), and view it as the Hamiltonian dynamics of a classical Hamiltonian system. We show that its quantisation describes $N$-body quantum mechanics, and that the "semi-classical" limit corresponding to $N^{-1} \rightarrow 0$ takes us back to the Hartree dynamics.

We sketch the key ideas behind our strategy.

(1) Use the Schwinger-Dyson expansion to construct the Heisenberg-picture dynamics of $p$-particle operators

$$
\mathrm{e}^{\mathrm{i} t H_{N}} \widehat{\mathrm{A}}_{N}\left(a^{(p)}\right) \mathrm{e}^{-\mathrm{i} t H_{N}}
$$

(in the notation of Sect. 3).

(2) Use Kato smoothing plus combinatorial estimates (counting of graphs) to prove convergence of the Schwinger-Dyson expansion on $N$-particle Hilbert space, uniformly in $N$ and for small $|t|$. Diagrams containing $l$ loops yield a contribution of order $N^{-l}$. 
(3) Use Kato smoothing plus combinatorial estimates to prove convergence of the iterative solution of the Hartree equation, for small $|t|$.

(4) Show that the Wick quantisation of the series in (3) is equal to the series of tree diagrams in (2).

(5) Extend (2) and (3) to arbitrary times by using unitarity and conservation laws.

This paper is organised as follows. In Sect. 2 we show that the classical Newtonian mechanics of point particles is the second quantisation of Vlasov theory, the latter being the mean-field (or "classical") limit of the former. The bulk of the paper is devoted to a rigorous analysis of the mean-field limit of Bose gases. In Sect. 3 we recall some important concepts of quantum many-body theory and introduce a general formalism which is convenient when dealing with quantum gases. Section 4 contains an implementation of Step (1) above. The convergence of the Schwinger-Dyson series for bounded interaction potentials is briefly discussed in Sect. 5. Section 6 implements Step (2) above. Steps (3), (4) and (5) are implemented in Sect. 7. Finally, Sect. 8 extends our results to more general interaction potentials as well as nonvanishing external potentials.

\section{Mean-Field Limit in Classical Mechanics}

In this section we consider the example of classical Newtonian mechanics to illustrate how the atomistic constitution of matter arises by quantisation of a continuum theory. The aim of this section is to give a brief and nonrigorous overview of some ideas that we shall develop in the context of quantum Bose gases, in full detail, in the following sections.

A classical gas is described as a continuous medium whose state is given by a nonnegative mass density $\mathrm{d} \mu(x, v)=M f(x, v) \mathrm{d} x \mathrm{~d} v$ on the "one-particle" phase space $\mathbb{R}^{3} \times \mathbb{R}^{3}$. Here $M$ is the mass of one "mole" of gas; $\mu(A)$ is the mass of gas in the phase space volume $A \subset \mathbb{R}^{3} \times \mathbb{R}^{3}$. Let $\int \mathrm{d} x \mathrm{~d} v f(x, v)=v<\infty$ denote the number of "moles" of the gas, so that the total mass of the gas is $\mu\left(\mathbb{R}^{3} \times \mathbb{R}^{3}\right)=v M$. An example of an equation of motion for $f(x, v)$ is the Vlasov equation

$$
\partial_{t} f_{t}(x, v)=-\left(v \cdot \nabla_{x} f_{t}\right)(x, v)+\frac{1}{m}\left(\nabla V_{\mathrm{eff}}\left[f_{t}\right] \cdot \nabla_{v} f_{t}\right)(x, v),
$$

where $m$ is a constant with the dimension of a mass, $t$ denotes time, and

$$
V_{\mathrm{eff}}[f](x)=V(x)+\int \mathrm{d} y W(x-y) \int \mathrm{d} v f(y, v) .
$$

Here $V$ is the potential of external forces acting on the gas and $W$ is a (two-body) potential describing self-interactions of the gas.

The Vlasov equation arises as the mean-field limit of a classical Hamiltonian system of $n$ point particles of mass $m$, with trajectories $\left(x_{i}(t)\right)_{i=1}^{n}$, moving in an external potential $V$ and interacting through two-body forces with potential $N^{-1} W\left(x_{i}-x_{j}\right)$. Here $N$ is the inverse coupling constant. We interpret $N$ as “Avogadro's number", i.e. as the number of particles per "mole" of gas. Thus, $M=m N$ and $n=v N$. More precisely, it is well-known (see $[3,16]$ ) that, under some technical assumptions on $V$ and $W$,

$$
f_{t}(x, v)=\underset{n \rightarrow \infty}{\mathrm{w}^{*}-\lim _{n}} \frac{v}{n} \sum_{i=1}^{n} \delta\left(x-x_{i}(t)\right) \delta\left(v-\dot{x}_{i}(t)\right)
$$


exists for all times $t$ and is the (unique) solution of (2.1), provided that this holds at time $t=0$. Here, $f_{t}$ is viewed as an element of the dual space of continuous bounded functions.

Note that $n$ and $N$ are, a priori, unrelated objects. While $n$ is the number of particles in the classical Hamiltonian system, $N^{-1}$ is by definition the coupling constant. The mean-field limit is the limit $n \rightarrow \infty$ while keeping $n \propto N$; the proportionality constant is $v$.

It is of interest to note that the Vlasov dynamics (2.1) may be interpreted as a Hamiltonian dynamics on an infinite-dimensional affine phase space $\Gamma_{\text {Vlasov }}$. To see this, we write

$$
f(x, v)=\bar{\alpha}(x, v) \alpha(x, v),
$$

where $\bar{\alpha}(x, v), \alpha(x, v)$ are complex coordinates on $\Gamma_{\text {Vlasov }}$. For our purposes it is enough to say that $\Gamma_{\text {Vlasov }}$ is some dense subspace of $L^{2}\left(\mathbb{R}^{6}\right)$ (typically a weighted Sobolev space

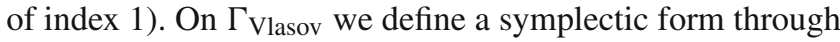

$$
\omega=\mathrm{i} \int \mathrm{d} x \mathrm{~d} v \mathrm{~d} \bar{\alpha}(x, v) \wedge \mathrm{d} \alpha(x, v) .
$$

This yields a Poisson bracket which reads

$$
\begin{aligned}
& \{\alpha(x, v), \alpha(y, w)\}=\{\bar{\alpha}(x, v), \bar{\alpha}(y, w)\}=0, \\
& \{\alpha(x, v), \bar{\alpha}(y, w)\}=\mathrm{i} \delta(x-y) \delta(v-w) .
\end{aligned}
$$

A Hamilton function $H$ is defined on $\Gamma_{\text {Vlasov }}$ through

$$
\begin{aligned}
H(\alpha):= & \mathrm{i} \int \mathrm{d} x \mathrm{~d} v \bar{\alpha}(x, v)\left[-v \cdot \nabla_{x}+\frac{1}{m} \nabla V(x) \cdot \nabla_{v}\right] \alpha(x, v) \\
& +\frac{\mathrm{i}}{m} \int \mathrm{d} x \mathrm{~d} v \bar{\alpha}(x, v)\left[\int \mathrm{d} y \mathrm{~d} w \nabla W(x-y)|\alpha(y, w)|^{2}\right] \cdot \nabla_{v} \alpha(x, v) .
\end{aligned}
$$

Note that $H$ is invariant under gauge transformations $\alpha \mapsto \mathrm{e}^{-\mathrm{i} \theta} \alpha, \bar{\alpha} \mapsto \mathrm{e}^{\mathrm{i} \theta} \bar{\alpha}$, which by Noether's theorem implies that $\int|\alpha|^{2} \mathrm{~d} x \mathrm{~d} v=\int f \mathrm{~d} x \mathrm{~d} v$ is conserved.

Let us abbreviate $K:=-\nabla V / m$ and $F:=-\nabla W / m$. After a short calculation using (2.3) we find that the Hamiltonian equation of motion $\dot{\alpha}_{t}(x, v)=\left\{H, \alpha_{t}(x, v)\right\}$ reads

$$
\begin{aligned}
\dot{\alpha}_{t}(x, v)= & \left(-v \cdot \nabla_{x}-K(x) \cdot \nabla_{v}\right) \alpha_{t}(x, v)-\int \mathrm{d} y \mathrm{~d} w F(x-y)\left|\alpha_{t}(y, w)\right|^{2} \cdot \nabla_{v} \alpha_{t}(x, v) \\
& +\int \mathrm{d} y \mathrm{~d} w F(x-y) \bar{\alpha}_{t}(y, w) \alpha_{t}(x, v) \cdot \nabla_{w} \alpha_{t}(y, w)
\end{aligned}
$$

Also, $\bar{\alpha}_{t}$ satisfies the complex conjugate equation. Therefore,

$$
\begin{aligned}
\frac{\mathrm{d}}{\mathrm{d} t}\left|\alpha_{t}(x, v)\right|^{2}= & \left(-v \cdot \nabla_{x}-K(x) \cdot \nabla_{v}\right)\left|\alpha_{t}(x, v)\right|^{2} \\
& -\int \mathrm{d} y \mathrm{~d} w F(x-y)\left|\alpha_{t}(y, w)\right|^{2} \cdot \nabla_{v}\left|\alpha_{t}(x, v)\right|^{2} \\
& +\left|\alpha_{t}(x, v)\right|^{2} \int \mathrm{d} y \mathrm{~d} w F(x-y) \\
& \cdot\left[\bar{\alpha}_{t}(y, w) \nabla_{w} \alpha_{t}(y, w)+\alpha_{t}(y, w) \nabla_{w} \bar{\alpha}_{t}(y, w)\right] .
\end{aligned}
$$


We assume that

$$
|\alpha(x, v)|=o\left(|(x, v)|^{-1}\right), \quad(x, v) \rightarrow \infty .
$$

We shall shortly see that this property is preserved under time-evolution. By integration by parts, we see that the second line of (2.6) vanishes, and we recover the Vlasov equation of motion (2.1) for $f=|\alpha|^{2}$.

We comment briefly on the existence and uniqueness of solutions to the Hamiltonian equation of motion (2.5). Following Braun and Hepp [3], we assume that $K$ and $F$ are bounded and continuously differentiable with bounded derivatives. We use polar coordinates

$$
\alpha=\beta \mathrm{e}^{\mathrm{i} \varphi},
$$

where $\varphi \in \mathbb{R}$ and $\beta \geq 0$. Then the Hamiltonian equation of motion (2.5) reads

$$
\begin{aligned}
\dot{\beta}_{t}(x, v)= & \left(-v \cdot \nabla_{x}-K(x) \cdot \nabla_{v}\right) \beta_{t}(x, v) \\
& -\int \mathrm{d} y \mathrm{~d} w F(x-y) \beta_{t}^{2}(y, w) \cdot \nabla_{v} \beta_{t}(x, v), \\
\dot{\varphi}_{t}(x, v)= & \left(-v \cdot \nabla_{x}-K(x) \cdot \nabla_{v}\right) \varphi_{t}(x, v)-\int \mathrm{d} y \mathrm{~d} w F(x-y) \beta_{t}^{2}(y, w) \cdot \nabla_{v} \varphi_{t}(x, v) \\
& +\int \mathrm{d} y \mathrm{~d} w F(x-y) \beta_{t}^{2}(y, w) \cdot \nabla_{w} \varphi_{t}(y, w) .
\end{aligned}
$$

We consider two cases.

(i) $\varphi=0$. In this case $\alpha=\beta$ and the equations of motion (2.8) are equivalent to the Vlasov equation for $f=\beta^{2}$. The results of $[3,16]$ then yield a global well-posedness result.

(ii) $\varphi \neq 0$. The equation of motion (2.8a) is independent of $\varphi$. Case (i) implies that it has a unique global solution. In order to solve the linear equation (2.8b), we apply a contraction mapping argument. Consider the space $X:=\left\{\varphi \in C\left(\mathbb{R}^{6}\right): \nabla \varphi \in\right.$ $\left.L^{\infty}\left(\mathbb{R}^{6}\right)\right\}$. Using Sobolev inequalities one finds that $X$, equipped with the norm $\|\varphi\|_{X}:=|\varphi(0)|+\|\nabla \varphi\|_{\infty}$, is a Banach space. We rewrite (2.8b) as an integral equation, and using standard methods show that, for small times, it has a unique solution. Using conservation of $\int \mathrm{d} x \mathrm{~d} v \beta_{t}^{2}$ we iterate this procedure to find a global solution. We omit further details.

Note that, as shown in [3], the solution $\beta_{t}$ can be written using a flow $\phi^{t}$ on the one-particle phase space: $\beta_{t}(x, v)=\beta_{0}\left(\phi^{-t}(x, v)\right)$. The flow $\phi^{t}(x, v)=(x(t), v(t))$ satisfies

$$
\begin{gathered}
\dot{x}(t)=v(t), \\
\dot{v}(t)=K(x(t))+\int \mathrm{d} y \mathrm{~d} w \beta_{t}^{2}(y, w) F(x(t)-y) .
\end{gathered}
$$

Using conservation of $\int \mathrm{d} x \mathrm{~d} v \beta_{t}^{2}$ we find that there is a constant $C$ such that $\left|\phi^{-t}(x, v)\right| \leq$ $|(x, v)|(1+t)+C\left(1+t^{2}\right)$. Therefore (2.7) holds for all times $t$ provided that it holds at time $t=0$.

The Hamiltonian formulation of Vlasov dynamics can serve as a starting point to recover the atomistic Hamiltonian mechanics of point particles by quantisation: Replace

$$
\bar{\alpha}(x, v) \rightarrow \widehat{\alpha}_{N}^{*}(x, v), \quad \alpha(x, v) \rightarrow \widehat{\alpha}_{N}(x, v),
$$


where $\widehat{\alpha}_{N}^{*}$ and $\widehat{\alpha}_{N}$ are creation and annihilation operators acting on the bosonic Fock space $\mathcal{F}_{+}\left(L^{2}\left(\mathbb{R}^{6}\right)\right)$; see Appendix A. They satisfy the canonical commutation relations (A.2); explicitly,

$$
\begin{aligned}
& {\left[\widehat{\alpha}_{N}(x, v), \widehat{\alpha}_{N}(y, w)\right]=\left[\widehat{\alpha}_{N}^{*}(x, v), \widehat{\alpha}_{N}^{*}(y, w)\right]=0,} \\
& {\left[\widehat{\alpha}_{N}(x, v), \widehat{\alpha}_{N}^{*}(y, w)\right]=\frac{1}{N} \delta(x-y) \delta(v-w) .}
\end{aligned}
$$

Given a function $A$ on $\Gamma_{\text {Vlasov }}$ which is a polynomial in $\bar{\alpha}$ and $\alpha$, we define an operator $\widehat{A}_{N}$ on $\mathcal{F}_{+}$by replacing $\alpha^{\#}$ with $\widehat{\alpha}_{N}^{\#}$ and Wick-ordering the resulting expression. We denote this quantisation map by $\widehat{(\cdot)}_{N}$. Here, $N^{-1}$ is the deformation parameter of the quantisation: We find that

$$
\left.\left[\widehat{A}_{N}, \widehat{B}_{N}\right]=\frac{N^{-1}}{\mathrm{i}} \widehat{\{A, B}\right\}_{N}+O\left(N^{-2}\right),
$$

for $N \rightarrow \infty$. Here $A$ and $B$ are polynomial functions on $\Gamma_{\text {Vlasov }}$.

The dynamics of a state $\Phi \in \mathcal{F}$ is given by the Schrödinger equation

$$
\mathrm{i} N^{-1} \partial_{t} \Phi_{t}=\widehat{H}_{N} \Phi_{t},
$$

where $\widehat{H}_{N}$ is the quantisation of the Vlasov Hamiltonian $H$. In order to identify the dynamics given by (2.10) with the classical dynamics of point particles, we study wave functions $\Phi^{(n)}\left(x_{1}, v_{1}, \ldots, x_{n}, v_{n}\right)$ in the $n$-particle sector of $\mathcal{F}_{+}$, and interpret $\rho^{(n)}:=$ $|\Phi|^{2}$ as a probability density on the $n$-body classical phase space. If $\Omega \in \mathcal{F}_{+}$denotes the vacuum vector annihilated by $\widehat{\alpha}_{N}(x, v)$ then

$\Phi^{(n)}=\frac{N^{n / 2}}{\sqrt{n !}} \int \mathrm{d} x_{1} \mathrm{~d} v_{1} \cdots \mathrm{d} x_{n} \mathrm{~d} v_{n} \Phi^{(n)}\left(x_{1}, v_{1}, \ldots, x_{n}, v_{n}\right) \widehat{\alpha}_{N}^{*}\left(x_{n}, v_{n}\right) \cdots \widehat{\alpha}_{N}^{*}\left(x_{1}, v_{1}\right) \Omega$.

It is a simple matter to check that (2.9) and (2.10) imply that

$\partial_{t} \Phi_{t}^{(n)}=\sum_{i=1}^{n}\left[-v_{i} \cdot \nabla_{x_{i}}+\frac{1}{m} \nabla V\left(x_{i}\right) \cdot \nabla_{v_{i}}\right] \Phi_{t}^{(n)}+\frac{1}{N} \sum_{1 \leq i \neq j \leq n} \frac{1}{m} \nabla W\left(x_{i}-x_{j}\right) \cdot \nabla_{v_{i}} \Phi_{t}^{(n)}$.

Also, $\overline{\Phi_{t}^{(n)}}$ satisfies the same equation. Therefore,

$\partial_{t} \rho_{t}^{(n)}=\sum_{i=1}^{n}\left[-v_{i} \cdot \nabla_{x_{i}}+\frac{1}{m} \nabla V\left(x_{i}\right) \cdot \nabla_{v_{i}}\right] \rho_{t}^{(n)}+\frac{1}{N} \sum_{1 \leq i \neq j \leq n} \frac{1}{m} \nabla W\left(x_{i}-x_{j}\right) \cdot \nabla_{v_{i}} \rho_{t}^{(n)}$.

This is the Liouville equation corresponding to the Hamiltonian equations of motion of $n$ classical point particles,

$$
\begin{aligned}
\partial_{t} x_{i} & =v_{i}, \\
m \partial_{t} v_{i} & =-\nabla V\left(x_{i}\right)-\frac{1}{N} \sum_{j \neq i} \nabla W\left(x_{i}-x_{j}\right) .
\end{aligned}
$$

Analogous results can be proven if $\widehat{\alpha}_{N}^{*}$ and $\widehat{\alpha}_{N}$ are chosen to be fermionic creation and annihilation operators obeying the canonical anti-commutation relations and acting on the fermionic Fock space $\mathcal{F}_{-}\left(L^{2}\left(\mathbb{R}^{6}\right)\right)$. 


\section{Quantum Gases: The Setup}

Although our main results are restricted to bosons, all of the following rather general formalism remains unchanged for fermions. We therefore consider both bosonic and fermionic statistics throughout Sects. 3 - 6. Details on systems of fermions will appear elsewhere.

Throughout the following we consider the one-particle Hilbert space

$$
\mathcal{H}:=L^{2}\left(\mathbb{R}^{3}, \mathrm{~d} x\right) .
$$

We refer the reader to Appendix A for our choice of notation and a short discussion of many-body quantum mechanics.

In the following a central role is played by the $p$-particles operators, i.e. closed operators $a^{(p)}$ on $\mathcal{H}_{ \pm}^{(p)}=P_{ \pm} \mathcal{H}^{\otimes p}$, where $P_{+}$and $P_{-}$denote symmetrisation and anti-symmetrisation, respectively. When using second-quantised notation it is convenient to use the operator kernel of $a^{(p)}$. Here is what this means (see [18] for details): Let $\mathscr{S}\left(\mathbb{R}^{d}\right)$ be the usual Schwartz space of smooth functions of rapid decrease, and $\mathscr{S}^{\prime}\left(\mathbb{R}^{d}\right)$ its topological dual. The nuclear theorem states that to every operator $A$ on $L^{2}\left(\mathbb{R}^{d}\right)$, such that the map $(f, g) \mapsto\langle f, A g\rangle$ is separately continuous on $\mathscr{S}\left(\mathbb{R}^{d}\right) \times \mathscr{S}\left(\mathbb{R}^{d}\right)$, there belongs a tempered distribution ("kernel") $\tilde{A} \in \mathscr{S}^{\prime}\left(\mathbb{R}^{2 d}\right)$, such that

$$
\langle f, A g\rangle=\tilde{A}(\bar{f} \otimes g) .
$$

In the following we identify $\tilde{A}$ with $A$. In the suggestive physicist's notation we thus have

$$
\begin{aligned}
\left\langle f^{(p)}, a^{(p)} g^{(p)}\right\rangle= & \frac{\int \mathrm{d} x_{1} \cdots \mathrm{d} x_{p} \mathrm{~d} y_{1} \cdots \mathrm{d} y_{p}}{} \overline{f^{(p)}}\left(x_{1}, \ldots, x_{p}\right) a^{(p)}\left(x_{1}, \ldots, x_{p} ; y_{1}, \ldots, y_{p}\right) g^{(p)}\left(y_{1}, \ldots, y_{p}\right),
\end{aligned}
$$

where $f, g \in \mathscr{S}\left(\mathbb{R}^{3 p}\right)$. It will be easy to verify that all $p$-particle operators that appear in the following satisfy the above condition; this is for instance the case for all bounded $a^{(p)} \in \mathcal{B}\left(\mathcal{H}^{\otimes p}\right)$.

Next, we define second quantisation $\widehat{\mathrm{A}}_{N}$. It maps a closed operator on $\mathcal{H}_{ \pm}^{(p)}$ to a closed operator on $\mathcal{F}_{ \pm}$according to the formula

$$
\begin{aligned}
\widehat{\mathrm{A}}_{N}\left(a^{(p)}\right):= & \int \mathrm{d} x_{1} \cdots \mathrm{d} x_{p} \mathrm{~d} y_{1} \cdots \mathrm{d} y_{p} \\
& \widehat{\psi}_{N}^{*}\left(x_{p}\right) \cdots \widehat{\psi}_{N}^{*}\left(x_{1}\right) a^{(p)}\left(x_{1}, \ldots, x_{p} ; y_{1}, \ldots, y_{p}\right) \widehat{\psi}_{N}\left(y_{1}\right) \cdots \widehat{\psi}_{N}\left(y_{p}\right) .
\end{aligned}
$$

Here $\widehat{\psi}_{N}^{\#}:=\frac{1}{\sqrt{N}} \widehat{\psi}^{\#}$, where $\widehat{\psi}^{\#}$ is the usual creation or annihilation operator; see Appendix A.

In order to understand the action of $\widehat{\mathrm{A}}_{N}\left(a^{(p)}\right)$ on $\mathcal{H}_{ \pm}^{(n)}$, we write

$$
\Phi^{(n)}=\frac{N^{n / 2}}{\sqrt{n !}} \int \mathrm{d} z_{1} \cdots \mathrm{d} z_{n} \Phi^{(n)}\left(z_{1}, \ldots, z_{n}\right) \widehat{\psi}_{N}^{*}\left(z_{n}\right) \cdots \widehat{\psi}_{N}^{*}\left(z_{1}\right) \Omega
$$


and apply $\widehat{\mathrm{A}}_{N}\left(a^{(p)}\right)$ to the right side. By using the (anti) commutation relations (A.2) to pull the $p$ annihilation operators $\widehat{\psi}_{N}\left(y_{i}\right)$ through the $n$ creation operators $\widehat{\psi}_{N}^{*}\left(z_{i}\right)$, and $\widehat{\psi}_{N}(x) \Omega=0$, we get the "first quantised" expression

$$
\left.\widehat{\mathrm{A}}_{N}\left(a^{(p)}\right)\right|_{\mathcal{H}_{ \pm}^{(n)}}= \begin{cases}\frac{p !}{N^{p}}\left(\begin{array}{l}
n \\
p
\end{array}\right) P_{ \pm}\left(a^{(p)} \otimes \mathbb{1}^{(n-p)}\right) P_{ \pm}, & n \geq p \\
0, & n<p\end{cases}
$$

This may be viewed as an alternative definition of $\widehat{\mathrm{A}}_{N}\left(a^{(p)}\right)$.

We define $\widehat{\mathfrak{A}}$ as the linear span of $\left\{\widehat{\mathrm{A}}_{N}\left(a^{(p)}\right): p \in \mathbb{N}, a^{(p)} \in \mathcal{B}\left(\mathcal{H}_{ \pm}^{(p)}\right)\right\}$. Then $\widehat{\mathfrak{A}}$ is a $*$-algebra of closable operators on $\mathcal{F}_{ \pm}^{0}$ (see Appendix A). We list some of its important properties, whose straightforward proofs we omit.

(i) $\widehat{\mathrm{A}}\left(a^{(p)}\right)^{*}=\widehat{\mathrm{A}}\left(\left(a^{(p)}\right)^{*}\right)$.

(ii) If $a^{(p)} \in \mathcal{B}\left(\mathcal{H}_{ \pm}^{(p)}\right)$ and $b^{(q)} \in \mathcal{B}\left(\mathcal{H}_{ \pm}^{(q)}\right)$, then

$$
\widehat{\mathrm{A}}_{N}\left(a^{(p)}\right) \widehat{\mathrm{A}}_{N}\left(b^{(q)}\right)=\sum_{r=0}^{\min (p, q)}\left(\begin{array}{l}
p \\
r
\end{array}\right)\left(\begin{array}{l}
q \\
r
\end{array}\right) \frac{r !}{N^{r}} \widehat{\mathrm{A}}_{N}\left(a^{(p)} \bullet r b^{(q)}\right),
$$

where

$$
a^{(p)} \bullet b^{(q)}:=P_{ \pm}\left(a^{(p)} \otimes \mathbb{1}^{(q-r)}\right)\left(\mathbb{1}^{(p-r)} \otimes b^{(q)}\right) P_{ \pm} \in \mathcal{B}\left(\mathcal{H}_{ \pm}^{(p+q-r)}\right) .
$$

(iii) The operator $\widehat{\mathrm{A}}\left(a^{(p)}\right)$ leaves the $n$-particle subspaces $\mathcal{H}_{ \pm}^{(n)}$ invariant.

(iv) If $a^{(p)} \in \mathcal{B}\left(\mathcal{H}_{ \pm}^{(p)}\right)$ and $b \in \mathcal{B}(\mathcal{H})$ is invertible, then

$$
\Gamma\left(b^{-1}\right) \widehat{\mathrm{A}}_{N}\left(a^{(p)}\right) \Gamma(b)=\widehat{\mathrm{A}}_{N}\left(\left(b^{-1}\right)^{\otimes p} a^{(p)} b^{\otimes p}\right),
$$

where $\Gamma(b)$ is defined on $\mathcal{H}_{ \pm}^{(n)}$ by $b^{\otimes n}$.

(v) If $a^{(p)} \in \mathcal{B}\left(\mathcal{H}_{ \pm}^{(p)}\right)$ then

$$
\left\|\left.\widehat{\mathrm{A}}_{N}\left(a^{(p)}\right)\right|_{\mathcal{H}_{ \pm}^{(n)}}\right\| \leq\left(\frac{n}{N}\right)^{p}\left\|a^{(p)}\right\|
$$

Of course, on an appropriate dense domain, (3.3) holds for unbounded operators $a^{(p)}$ and $b^{(q)}$ too. We introduce the notation

$$
\left[a^{(p)}, b^{(q)}\right]_{r}:=a^{(p)} \bullet_{r} b^{(q)}-b^{(q)} \bullet_{r} a^{(p)} .
$$

Note that $\left[a^{(p)}, b^{(q)}\right]_{0}=0$. Thus,

$$
\left[\widehat{\mathrm{A}}_{N}\left(a^{(p)}\right), \widehat{\mathrm{A}}_{N}\left(b^{(q)}\right)\right]=\sum_{r=1}^{\min (p, q)}\left(\begin{array}{l}
p \\
r
\end{array}\right)\left(\begin{array}{l}
q \\
r
\end{array}\right) \frac{r !}{N^{r}} \widehat{\mathrm{A}}_{N}\left(\left[a^{(p)}, b^{(q)}\right]_{r}\right)
$$

We now move on to discuss dynamics. Take a one-particle Hamiltonian $h^{(1)} \equiv h$ of the form $h=-\Delta+v$, where $\Delta$ is the Laplacian over $\mathbb{R}^{3}$ and $v$ is some real function. We denote by $V$ the multiplication operator $v(x)$. Two-body interactions are described 
by a real, even function $w$ on $\mathbb{R}^{3}$. This induces a two-particle operator $W^{(2)} \equiv W$ on $\mathcal{H}^{\otimes 2}$, defined as the multiplication operator $w\left(x_{1}-x_{2}\right)$. We define the Hamiltonian

$$
\widehat{H}_{N}:=\widehat{\mathrm{A}}_{N}(h)+\frac{1}{2} \widehat{\mathrm{A}}_{N}(W) .
$$

Under suitable assumptions on $v$ and $w$ that we make precise in the following sections, one shows that $\widehat{H}_{N}$ is a well-defined self-adjoint operator on $\mathcal{F}_{ \pm}$. It is convenient to introduce $H_{N}:=N \widehat{H}_{N}$. On $\mathcal{H}_{ \pm}^{(n)}$ we have the "first quantised" expression

$$
\left.H_{N}\right|_{\mathcal{H}_{ \pm}^{(n)}}=\sum_{i=1}^{n} h_{i}+\frac{1}{N} \sum_{1 \leq 1<j \leq n} W_{i j}=: H_{0}+\frac{1}{N} W
$$

in self-explanatory notation.

\section{Schwinger-Dyson Expansion and Loop Counting}

Without loss of generality, we assume throughout the following that $t \geq 0$.

Let $a^{(p)} \in \mathcal{B}\left(\mathcal{H}_{ \pm}^{(p)}\right)$ and $w$ be bounded, i.e. $w \in L^{\infty}\left(\mathbb{R}^{3}\right)$. Using the fundamental theorem of calculus and the fact that the unitary group $\left(\mathrm{e}^{-\mathrm{i} t H_{0}}\right)_{t \in \mathbb{R}}$ is strongly differentiable one finds

$$
\begin{aligned}
\mathrm{e}^{\mathrm{i} t H_{N}} & \widehat{\mathrm{A}}_{N}\left(a^{(p)}\right) \mathrm{e}^{-\mathrm{i} t H_{N}} \Phi^{(n)} \\
& =\left.\mathrm{e}^{\mathrm{i} s H_{N}} \mathrm{e}^{-\mathrm{i} t s H_{0}} \mathrm{e}^{\mathrm{i} t H_{0}} \widehat{\mathrm{A}}_{N}\left(a^{(p)}\right) \mathrm{e}^{-\mathrm{i} t H_{0}} \mathrm{e}^{\mathrm{i} s H_{0}} \mathrm{e}^{-\mathrm{i} s H_{N}} \Phi^{(n)}\right|_{s=t} \\
& =\widehat{\mathrm{A}}_{N}\left(a_{t}^{(p)}\right) \Phi^{(n)}+\int_{0}^{t} \mathrm{~d} s \mathrm{e}^{\mathrm{i} s H_{N}} \mathrm{e}^{-\mathrm{i} s H_{0}} \frac{\mathrm{i} N}{2}\left[\widehat{\mathrm{A}}_{N}\left(W_{s}\right), \widehat{\mathrm{A}}_{N}\left(a_{t}^{(p)}\right)\right] \mathrm{e}^{\mathrm{i} s H_{0}} \mathrm{e}^{-\mathrm{i} s H_{N}} \Phi^{(n)},
\end{aligned}
$$

where $(\cdot)_{t}:=\Gamma\left(\mathrm{e}^{\mathrm{i} t h}\right)(\cdot) \Gamma\left(\mathrm{e}^{-\mathrm{i} t h}\right)$ denotes free time evolution. As an equation between operators defined on $\mathcal{F}_{ \pm}^{0}$, this reads

$$
\begin{aligned}
& \mathrm{e}^{\mathrm{i} t H_{N}} \widehat{\mathrm{A}}_{N}\left(a^{(p)}\right) \mathrm{e}^{-\mathrm{i} t H_{N}} \\
& \quad=\widehat{\mathrm{A}}_{N}\left(a_{t}^{(p)}\right)+\int_{0}^{t} \mathrm{~d} s \mathrm{e}^{\mathrm{i} s H_{N}} \mathrm{e}^{-\mathrm{i} s H_{0}} \frac{\mathrm{i} N}{2}\left[\widehat{\mathrm{A}}_{N}\left(W_{s}\right), \widehat{\mathrm{A}}_{N}\left(a_{t}^{(p)}\right)\right] \mathrm{e}^{\mathrm{i} s H_{0}} \mathrm{e}^{-\mathrm{i} s H_{N}} .
\end{aligned}
$$

Iteration of (4.1) yields the formal power series

$$
\sum_{k=0}^{\infty} \int_{\Delta^{k}(t)} \mathrm{d} t \frac{(\mathrm{i} N)^{k}}{2^{k}}\left[\widehat{\mathrm{A}}_{N}\left(W_{t_{k}}\right), \ldots\left[\widehat{\mathrm{A}}_{N}\left(W_{t_{1}}\right), \widehat{\mathrm{A}}_{N}\left(a_{t}^{(p)}\right)\right] \ldots\right] .
$$

It is easy to see that, on $\mathcal{H}_{ \pm}^{(n)}$, the $k^{\text {th }}$ term of (4.2) is bounded in norm by

$$
\frac{\left(t n^{2}\|w\|_{\infty} / N\right)^{k}}{k !}\left(\frac{n}{N}\right)^{p}\left\|a^{p}\right\| .
$$

Therefore, on $\mathcal{H}_{ \pm}^{(n)}$, the series (4.2) converges in norm for all times. Furthermore, (4.3) implies that the rest term arising from the iteration of (4.1) vanishes for $k \rightarrow \infty$, so that (4.2) is equal to (4.1). 


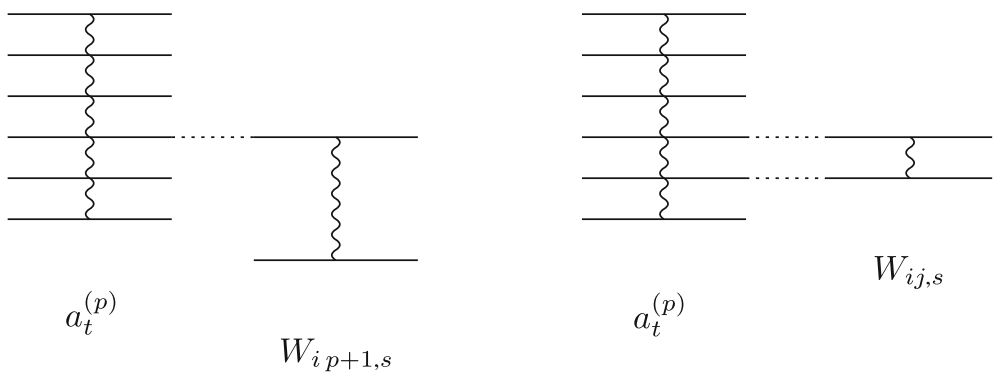

Fig. 4.1. Two terms of the product $\widehat{\mathrm{A}}_{N}\left(a_{t}^{(p)}\right) \widehat{\mathrm{A}}_{N}\left(W_{S}\right)$, represented as labelled diagrams. A tree term (left) produces a tree diagram. A loop term (right) produces a diagram with one loop

The mean-field limit is the limit $n=v N \rightarrow \infty$, where $v>0$ is some constant. The above estimate is clearly inadequate to prove statements about the mean-field limit. In order to obtain estimates uniform in $N$, more care is needed.

To see why the above estimate is so crude, consider the commutator

$$
\left.\frac{\mathrm{i} N}{2}\left[\widehat{\mathrm{A}}_{N}\left(W_{s}\right), \widehat{\mathrm{A}}_{N}\left(a_{t}^{(p)}\right)\right]\right|_{\mathcal{H}_{ \pm}^{(n)}}=\frac{p !}{N^{p}}\left(\begin{array}{l}
n \\
p
\end{array}\right) \frac{\mathrm{i}}{N} P_{ \pm} \sum_{1 \leq i<j \leq n}\left[W_{i j, s}, a_{t}^{(p)} \otimes \mathbb{1}^{(n-p)}\right] P_{ \pm} .
$$

We see that most terms of the commutator vanish (namely, whenever $p<i<j$ ). Thus, for large $n$, the above estimates are highly wasteful. This can be remedied by more careful bookkeeping. We split the commutator into two terms: the tree terms, defined by $1 \leq i \leq p$ and $p+1 \leq j \leq n$, and the loop terms, defined by $1 \leq i<j \leq p$. All other terms vanish. This splitting can also be inferred from (3.8).

The naming originates from a diagrammatic representation (see Fig. 4.1). A $p$-particle operator is represented as a wiggly vertical line to which are attached $p$ horizontal branches on the left and $p$ horizontal branches on the right. Each branch on the left represents a creation operator $\widehat{\psi}_{N}^{*}\left(x_{i}\right)$, and each branch on the right an annihilation operator $\widehat{\psi}_{N}\left(y_{i}\right)$. The product $\widehat{\mathrm{A}}_{N}\left(a^{(p)}\right) \widehat{\mathrm{A}}_{N}\left(b^{(q)}\right)$ of two operators is given by the sum over all possible pairings of the annihilation operators in $\widehat{\mathrm{A}}_{N}\left(a^{(p)}\right)$ with the creation operators in $\widehat{\mathrm{A}}_{N}\left(b^{(q)}\right)$. Such a contraction is graphically represented as a horizontal line joining the corresponding branches. We consider diagrams that arise in this manner from the multiplication of a finite number of operators of the form $\widehat{\mathrm{A}}_{N}\left(a^{(p)}\right)$.

We now generalise this idea to a systematic scheme for the multiple commutators appearing in the Schwinger-Dyson expansion. To this end, we decompose the multiple commutator

$$
\frac{(\mathrm{i} N)^{k}}{2^{k}}\left[\widehat{\mathrm{A}}_{N}\left(W_{t_{k}}\right), \ldots\left[\widehat{\mathrm{A}}_{N}\left(W_{t_{1}}\right), \widehat{\mathrm{A}}_{N}\left(a_{t}^{(p)}\right)\right] \ldots\right]
$$

into a sum of $2^{k}$ terms obtained by writing out each commutator. Each resulting term is a product of $k+1$ second-quantised operators, which we furthermore decompose into a sum over all possible contractions for which $r>0$ in (3.3) (at least one contraction for each multiplication). The restriction $r>0$ follows from $\left[a^{(p)}, b^{(q)}\right]_{0}=0$. This is equivalent to saying that all diagrams are connected. 
We call the resulting terms elementary. The idea is to classify all elementary terms according to their number of loops $l$. Write

$$
\frac{(\mathrm{i} N)^{k}}{2^{k}}\left[\widehat{\mathrm{A}}_{N}\left(W_{t_{k}}\right), \ldots\left[\widehat{\mathrm{A}}_{N}\left(W_{t_{1}}\right), \widehat{\mathrm{A}}_{N}\left(a_{t}^{(p)}\right)\right] \ldots\right]=\sum_{l=0}^{k} \frac{1}{N^{l}} \widehat{\mathrm{A}}_{N}\left(G_{t, t_{1}, \ldots, t_{k}}^{(k, l)}\left(a^{(p)}\right)\right),
$$

where $G_{t, t_{1}, \ldots, t_{k}}^{(k, l)}\left(a^{(p)}\right)$ is a $(p+k-l)$-particle operator, equal to the sum of all elementary terms with $l$ loops. It is defined through the recursion relation (on $\mathcal{H}_{ \pm}^{(p+k-l)}$ )

$$
\begin{aligned}
G_{t, t_{1}, \ldots, t_{k}}^{(k, l)}\left(a^{(p)}\right)= & \mathrm{i}(p+k-l-1)\left[W_{t_{k}}, G_{t, t_{1}, \ldots, t_{k-1}}^{(k-1, l)}\left(a^{(p)}\right)\right]_{1} \\
& +\mathrm{i}\left(\begin{array}{c}
p+k-l \\
2
\end{array}\right)\left[W_{t_{k}}, G_{t, t_{1}, \ldots, t_{k-1}}^{(k-1, l-1)}\left(a^{(p)}\right)\right]_{2} \\
= & \mathrm{i} P_{ \pm} \sum_{i=1}^{p+k-l-1}\left[W_{i p+k-l, t_{k}}, G_{t, t_{1}, \ldots, t_{k-1}}^{(k-1, l)}\left(a^{(p)}\right) \otimes \mathbb{1}\right] P_{ \pm} \\
& +\mathrm{i} P_{ \pm} \sum_{1 \leq i<j \leq p+k-l}\left[W_{i j, t_{k}}, G_{t, t_{1}, \ldots, t_{k-1}}^{(k-1, l-1)}\left(a^{(p)}\right)\right] P_{ \pm},
\end{aligned}
$$

as well as $G_{t}^{(0,0)}\left(a^{(p)}\right):=a_{t}^{(p)}$. If $l<0, l>k$, or $p+k-l>n$, then $G_{t, t_{1}, \ldots, t_{k}}^{(k, l)}\left(a^{(p)}\right)=0$. The interpretation of the recursion relation is simple: a $(k, l)$-term arises from either a $(k-1, l)$-term without adding a loop or from a $(k-1, l-1)$-term to which a loop is added. It is not hard to see, using induction on $k$ and the definition (4.5), that (4.4) holds. It is often convenient to have an explicit formula for the decomposition into elementary terms:

$$
G_{t, t_{1}, \ldots, t_{k}}^{(k, l)}\left(a^{(p)}\right)=\sum_{\alpha=1}^{c(p, k, l)} G_{t, t_{1}, \ldots, t_{k}}^{(k, l)(\alpha)}\left(a^{(p)}\right)
$$

where $G_{t, t_{1}, \ldots, t_{k}}^{(k, l)\left(a^{(p)}\right)}$ is an elementary term, and $c(p, k, l)$ is the number of elementary terms in $G_{t, t_{1}, \ldots, t_{k}}^{(k, l)}\left(a^{(p)}\right)$.

In order to establish a one-to-one correspondence between elementary terms and diagrams, we introduce a labelling scheme for diagrams. Consider an elementary term arising from a choice of contractions in the multiple commutator of order $k$, along with its diagram. We label all vertical lines $v$ with an index $i_{v} \in \mathbb{N}$ as follows. The vertical line of $a^{(p)}$ is labelled by 0 . The vertical line of the first (i.e. innermost in the multiple commutator) interaction operator is labelled by 1 , of the second by 2 , and so on (see Fig. 4.2). Conversely, every elementary term is uniquely determined by its labelled diagram. We consequently use $\alpha=1, \ldots, c(p, k, l)$ to index either elementary terms or labelled diagrams.

Use the shorthand $t=\left(t_{1}, \ldots, t_{k}\right)$ and define

$$
G_{t}^{(k, l)}\left(a^{(p)}\right):=\int_{\Delta^{k}(t)} \mathrm{d} \underline{t} G_{t, \underline{t}}^{(k, l)}\left(a^{(p)}\right)
$$

In summary, we have an expansion in terms of the number of loops $l$ : 


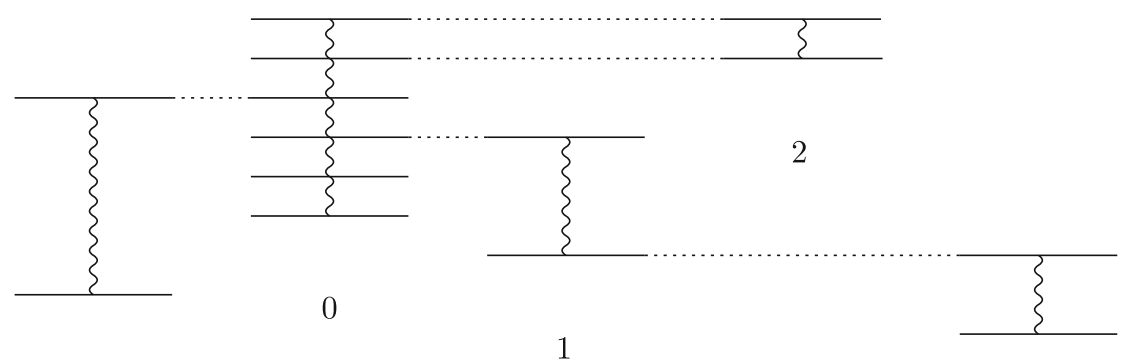

3

4

Fig. 4.2. The labelled diagram corresponding to a one-loop elementary term in the commutator of order 4

$$
\mathrm{e}^{\mathrm{i} t H_{N}} \widehat{\mathrm{A}}_{N}\left(a^{(p)}\right) \mathrm{e}^{-\mathrm{i} t H_{N}}=\sum_{k=0}^{\infty} \sum_{l=0}^{k} \frac{1}{N^{l}} \widehat{\mathrm{A}}_{N}\left(G_{t}^{(k, l)}\left(a^{(p)}\right)\right),
$$

which converges in norm on $\mathcal{H}_{ \pm}^{(n)}, n \in \mathbb{N}$, for all times $t$.

\section{Convergence for Bounded Interaction}

For a bounded interaction potential, $\|w\|_{\infty}<\infty$, it is now straightforward to control the mean-field limit.

Lemma 5.1. We have the bound

$$
\left\|G_{t, \underline{t}}^{(k, l)}\left(a^{(p)}\right)\right\| \leq c(p, k, l)\|w\|_{\infty}^{k}\left\|a^{(p)}\right\|
$$

Furthermore,

$$
c(p, k, l) \leq 2^{k}\left(\begin{array}{l}
k \\
l
\end{array}\right)(p+k-l)^{l}(p+k-1) \cdots p .
$$

Proof. Assume first that $l=0$. Then the number of labelled diagrams is clearly given by $2^{k} p \cdots(p+k-1)$. Now if there are $l$ loops, we may choose to add them at any $l$ of the $k$ steps when computing the multiple commutator. Furthermore, each addition of a loop produces at most $p+k-l$ times more elementary terms than the addition of a tree branch. Combining these observations, we arrive at the claimed bound for $c(p, k, l)$.

Alternatively, it is a simple exercise to show the claim, with $c(p, k, l)$ replaced by the bound (5.2), by induction on $k$.

Lemma 5.2. Let $v>0$ and $t<\left(8 v\|w\|_{\infty}\right)^{-1}$. Then, on $\mathcal{H}_{ \pm}^{(v N)}$, the Schwinger-Dyson series (4.7) converges in norm, uniformly in $N$. 
Proof. Recall that $p+k-l \leq n$ for nonvanishing $\left.\widehat{\mathrm{A}}_{N}\left(G_{t, \underline{t}}^{(k, l)}\left(a^{(p)}\right)\right)\right|_{\mathcal{H}_{ \pm}^{(n)}}$. Using the symbol $I_{\{A\}}$, defined as 1 if $A$ is true and 0 if $A$ is false, we find

$$
\begin{aligned}
& \sum_{k=0}^{\infty} \sum_{l=0}^{k} \frac{1}{N^{l}} \int_{\Delta^{k}(t)} \mathrm{d} \underline{t}\left\|\left.\widehat{\mathrm{A}}_{N}\left(G_{t, \underline{t}}^{(k, l)}\left(a^{(p)}\right)\right)\right|_{\mathcal{H}_{ \pm}^{(v N)}}\right\| \\
& \quad \leq \sum_{k=0}^{\infty} \sum_{l=0}^{k} \frac{(p+k-l)^{l}}{N^{l}} I_{\{p+k-l \leq v N\}} \frac{1}{k !}\left(2\|w\|_{\infty} t\right)^{k}\left(\begin{array}{c}
k \\
l
\end{array}\right)\left(\begin{array}{c}
p+k-1 \\
k
\end{array}\right) k ! v^{p+k-l}\left\|a^{(p)}\right\| \\
& \quad \leq \sum_{k=0}^{\infty}\left(8 v\|w\|_{\infty} t\right)^{k}(2 v)^{p}\left\|a^{(p)}\right\| \\
& \quad=\frac{1}{1-8 v\|w\|_{\infty} t}(2 v)^{p}\left\|a^{(p)}\right\|
\end{aligned}
$$

where we used that $\sum_{l=0}^{k}\left(\begin{array}{l}k \\ l\end{array}\right)=2^{k}$, and in particular $\left(\begin{array}{l}k \\ l\end{array}\right) \leq 2^{k}$.

In the spirit of semi-classical expansions, we can rewrite the Schwinger-Dyson series to get a " $1 / N$-expansion", whereby all $l$-loop terms add up to an operator of order $O\left(N^{-l}\right)$.

Lemma 5.3. Let $t<\left(8 v\|w\|_{\infty}\right)^{-1}$ and $L \in \mathbb{N}$. Then we have on $\mathcal{H}_{ \pm}^{(v N)}$,

$$
\mathrm{e}^{\mathrm{i} t H_{N}} \widehat{\mathrm{A}}_{N}\left(a^{(p)}\right) \mathrm{e}^{-\mathrm{i} t H_{N}}=\sum_{l=0}^{L-1} \frac{1}{N^{l}} \sum_{k=l}^{\infty} \widehat{\mathrm{A}}_{N}\left(G_{t}^{(k, l)}\left(a^{(p)}\right)\right)+O\left(\frac{1}{N^{L}}\right),
$$

where the sum converges uniformly in $N$.

Proof. Instead of the full Schwinger-Dyson expansion (4.2), we can stop the expansion whenever $L$ loops have been generated. More precisely, we iterate (4.1) and use (3.8) at each iteration to split the commutator into tree $(r=1)$ and loop $(r=2)$ terms. Whenever a term obtained in this fashion has accumulated $L$ loops, we stop expanding and put it into a remainder term. Thus all fully expanded terms are precisely those arising from diagrams containing up to $L-1$ loops, and it is not hard to show that the remainder term is of order $N^{-L}$.

In view of later applications, we also give a proof using the fully expanded SchwingerDyson series. From Lemma 5.2 we know that the sum converges on $\mathcal{H}_{ \pm}^{(v N)}$ in norm, uniformly in $N$, and can be reordered as

$$
\mathrm{e}^{\mathrm{i} t H_{N}} \widehat{\mathrm{A}}_{N}\left(a^{(p)}\right) \mathrm{e}^{-\mathrm{i} t H_{N}}=\sum_{l=0}^{\infty} \frac{1}{N^{l}} \sum_{k=l}^{\infty} \int_{\Delta^{k}(t)} \mathrm{d} t \widehat{\mathrm{A}}_{N}\left(G_{t, \underline{t}}^{(k, l)}\left(a^{(p)}\right)\right),
$$

as an identity on $\mathcal{H}_{ \pm}^{(v N)}$. Proceeding as above we find 


$$
\begin{aligned}
\sum_{l=L}^{\infty} & \frac{1}{N^{l}} \sum_{k=l}^{\infty} \int_{\Delta^{k}(t)} \mathrm{d} \underline{t}\left\|\left.\widehat{\mathrm{A}}_{N}\left(G_{t, \underline{t}}^{(k, l)}\left(a^{(p)}\right)\right)\right|_{\mathcal{H}_{ \pm}^{(v N)}}\right\| \\
\leq & \frac{1}{N^{L}} \sum_{l=L}^{\infty} \sum_{k=l}^{\infty} \frac{(p+k-l)^{l}}{N^{l-L}} I_{\{p+k-l \leq v N\}} \\
& \times \frac{1}{k !}\left(2\|w\|_{\infty} t\right)^{k}\left(\begin{array}{c}
k \\
l
\end{array}\right)\left(\begin{array}{c}
p+k-1 \\
k
\end{array}\right) k ! v^{p+k-l}\left\|a^{(p)}\right\| \\
\leq & \frac{1}{(v N)^{L}} \sum_{l=L}^{\infty} \sum_{k=l}^{\infty}(p+k-l)^{L}\left(8 v\|w\|_{\infty} t\right)^{k}(2 v)^{p}\left\|a^{(p)}\right\| \\
= & \frac{1}{(v N)^{L}} \sum_{l=L}^{\infty} \sum_{k=0}^{\infty}(p+k)^{L}\left(8 v\|w\|_{\infty} t\right)^{k+l}(2 v)^{p}\left\|a^{(p)}\right\| \\
\leq & \frac{1}{(v N)^{L}} \sum_{l=L}^{\infty}\left(8 v\|w\|_{\infty} t\right)^{l} \frac{\mathrm{e}^{p} L !}{\left(1-8 v\|w\|_{\infty} t\right)^{L+1}}(2 v)^{p}\left\|a^{(p)}\right\| \\
= & \frac{1}{(v N)^{L}} \frac{\mathrm{e}^{p} L !\left(8 v\|w\|_{\infty} t\right)^{L}}{\left(1-8 v\|w\|_{\infty} t\right)^{L+2}}(2 v)^{p}\left\|a^{(p)}\right\|,
\end{aligned}
$$

where we used that $\sum_{k=0}^{\infty}(p+k)^{L} x^{k} \leq \frac{\mathrm{e}^{p} L !}{(1-x)^{L+1}}$.

\section{Convergence for Coulomb Interaction}

In this section we consider an interaction potential of the form

$$
w(x)=\kappa \frac{1}{|x|},
$$

where $\kappa \in \mathbb{R}$. We take the one-body Hamiltonian to be

$$
h=-\Delta \text {, }
$$

the nonrelativistic kinetic energy without external potentials. We assume this form of $h$ and $w$ throughout Sects. 6 and 7. In Sect. 8, we discuss some generalisations.

6.1. Kato smoothing. The non-relativistic dispersive nature of the free time evolution $\mathrm{e}^{\mathrm{i} t \Delta}$ is essential for controlling singular potentials. The key tool for all of the following is the Kato smoothing estimate:

$$
\int_{\mathbb{R}}\left\||x|^{-1} \mathrm{e}^{\mathrm{i} t \Delta} \psi\right\|^{2} \mathrm{~d} t \leq \pi\|\psi\|^{2},
$$

where $\psi \in L^{2}\left(\mathbb{R}^{3}\right)$. Estimate (6.2) follows from Kato's theory of smooth perturbations; see $[20,23]$. In Sect. 8 we provide a proof of (6.2) (without the sharp constant $\pi$ ), for a larger class of interaction potentials, using Strichartz estimates.

In order to avoid tedious discussions of operator domains in equations such as (4.1), we introduce a cutoff to make the interaction potential bounded. For $\varepsilon \geq 0$ set

$$
w^{\varepsilon}(x):=w(x) I_{\left\{|w(x)| \leq \varepsilon^{-1}\right\}},
$$


so that $\left\|w^{\varepsilon}\right\|_{\infty} \leq \varepsilon^{-1}$. Now (6.2) implies, for $\varepsilon \geq 0$,

$$
\int_{\mathbb{R}}\left\|w^{\varepsilon} \mathrm{e}^{\mathrm{i} t \Delta} \psi\right\|^{2} \mathrm{~d} t \leq \int_{\mathbb{R}}\left\|w \mathrm{e}^{\mathrm{i} t \Delta} \psi\right\|^{2} \mathrm{~d} t \leq \pi \kappa^{2}\|\psi\|^{2} .
$$

An immediate consequence is the following lemma.

Lemma 6.1. Let $\Phi^{(n)} \in \mathcal{H}_{ \pm}^{(n)}$. Then

$$
\int_{\mathbb{R}}\left\|W_{i j}^{\varepsilon} \mathrm{e}^{-\mathrm{i} t H_{0}} \Phi^{(n)}\right\|^{2} \mathrm{~d} t \leq \frac{\pi \kappa^{2}}{2}\left\|\Phi^{(n)}\right\|^{2} .
$$

Proof. By symmetry we may assume that $(i, j)=(1,2)$. Choose centre of mass coordinates $X:=\left(x_{1}+x_{2}\right) / 2$ and $\xi=x_{2}-x_{1}$, set $\tilde{\Phi}^{(n)}\left(X, \xi, x_{3}, \ldots, x_{n}\right):=\Phi^{(n)}\left(x_{1}, \ldots, x_{n}\right)$, and write

$$
\int_{\mathbb{R}}\left\|W_{12}^{\varepsilon} \mathrm{e}^{-\mathrm{i} t H_{0}} \Phi^{(n)}\right\|^{2} \mathrm{~d} t=\int_{\mathbb{R}}\left\|w^{\varepsilon}(\xi) \mathrm{e}^{2 \mathrm{i} t \Delta_{\xi}} \tilde{\Phi}^{(n)}\right\|^{2} \mathrm{~d} t,
$$

since $H_{0}=-\Delta_{1}-\Delta_{2}=-\Delta_{X} / 2-2 \Delta_{\xi}$ and $\left[\Delta_{X}, w^{\varepsilon}(\xi)\right]=0$. Therefore, by (6.3) and Fubini's theorem, we find

$$
\begin{aligned}
& \int_{\mathbb{R}}\left\|W_{12}^{\varepsilon} \mathrm{e}^{-\mathrm{i} t H_{0}} \Phi^{(n)}\right\|^{2} \mathrm{~d} t \\
& \quad=\int \mathrm{d} X \mathrm{~d} x_{3} \cdots \mathrm{d} x_{n} \int \mathrm{d} t \mathrm{~d} \xi\left|w^{\varepsilon}(\xi) \mathrm{e}^{2 \mathrm{i} t \Delta_{\xi}} \tilde{\Phi}^{(n)}\left(X, \xi, x_{3}, \ldots, x_{n}\right)\right|^{2} \\
& \quad \leq \frac{\pi \kappa^{2}}{2} \int \mathrm{d} X \mathrm{~d} x_{3} \cdots \mathrm{d} x_{n} \int \mathrm{d} \xi\left|\tilde{\Phi}^{(n)}\left(X, \xi, x_{3}, \ldots, x_{n}\right)\right|^{2} \\
& =\frac{\pi \kappa^{2}}{2}\left\|\Phi^{(n)}\right\|^{2} .
\end{aligned}
$$

By Cauchy-Schwarz we then find that

$$
\int_{0}^{t}\left\|W_{i j, s}^{\varepsilon} \Phi^{(n)}\right\| \mathrm{d} s \leq t^{1 / 2}\left(\int_{\mathbb{R}}\left\|W_{i j}^{\varepsilon} \mathrm{e}^{-\mathrm{i} s H_{0}} \Phi^{(n)}\right\|^{2} \mathrm{~d} s\right)^{1 / 2} \leq\left(\frac{\pi \kappa^{2} t}{2}\right)^{1 / 2}\left\|\Phi^{(n)}\right\| .
$$

By iteration, this implies that, for all elementary terms $\alpha$,

$$
\int_{0}^{t} \mathrm{~d} t_{1} \ldots \int_{0}^{t} \mathrm{~d} t_{k}\left\|G_{t, \underline{t}}^{(k, l)(\alpha), \varepsilon}\left(a^{(p)}\right) \Phi^{(p+k-l)}\right\| \leq\left(\frac{\pi \kappa^{2} t}{2}\right)^{k / 2}\left\|a^{(p)}\right\|\left\|\Phi^{(p+k-l)}\right\|,
$$

where the superscript $\varepsilon$ reminds us that $G_{t, \underline{t}}^{(k, l)(\alpha), \varepsilon}\left(a^{(p)}\right)$ is computed with the regularised potential $w^{\varepsilon}$. Thus one finds

$$
\left\|G_{t}^{(k, l), \varepsilon}\left(a^{(p)}\right)\right\| \leq c(p, k, l)\left(\frac{\pi \kappa^{2} t}{2}\right)^{k / 2}\left\|a^{(p)}\right\|,
$$

for all $\varepsilon \geq 0$.

Unfortunately, the above procedure does not recover the factor $1 / k$ ! arising from the time-integration over the $k$-simplex $\Delta^{k}(t)$, which is essential for our convergence 
estimates. First iterating (6.4) and then using Cauchy-Schwarz yields a factor $1 / \sqrt{k !}$, which is still not good enough.

A solution to this problem must circumvent the highly wasteful procedure of replacing the integral over $\Delta^{k}(t)$ with an integral over $[0, t]^{k}$. The key observation is that, in the sum over all labelled diagrams, each diagram appears of the order of $k$ ! times with different labellings.

6.2. Graph counting. In order to make the above idea precise, we make use of graphs (related to the above diagrams) to index terms in our expansion of the multiple commutator

$$
\frac{(\mathrm{i} N)^{k}}{2^{k}}\left[\widehat{\mathrm{A}}_{N}\left(W_{t_{k}}\right), \ldots\left[\widehat{\mathrm{A}}_{N}\left(W_{t_{1}}\right), \widehat{\mathrm{A}}_{N}\left(a_{t}^{(p)}\right)\right] \ldots\right] .
$$

The idea is to assign to each second quantised operator a vertex $v=0, \ldots, k$, and to represent each creation and annihilation with an incident edge. A pairing of an annihilation operator with a creation operator is represented by joining the corresponding edges. The vertex 0 has $2 p$ edges and the vertices $1, \ldots, k$ have 4 edges. We call the vertex 0 the root.

The edges incident to each vertex $v$ are labelled using a pair $\lambda=(d, i)$, where $d=a, c$ is the direction ( $a$ stands for "annihilation" and $c$ for "creation") and $i$ labels edges of the same direction; $i=1, \ldots, p$ if $v=0$ and $i=1,2$ if $v=1, \ldots, k$. Thus, a labelled edge is of the form $\left\{\left(v_{1}, \lambda_{1}\right),\left(v_{2}, \lambda_{2}\right)\right\}$. Graphs $\mathcal{G}$ with such labelled edges are graphs over the vertex set $V(\mathcal{G})=\{(v, \lambda)\}$. We denote the set of edges of a graph $\mathcal{G}$ (a set of unordered pairs of vertices in $V(\mathcal{G})$ ) by $E(\mathcal{G})$. The degree of each $(v, \lambda)$ is either 0 or 1 ; we call $(v, \lambda)$ an empty edge of $v$ if its degree is 0 . We often speak of connecting two empty edges, as well as removing a nonempty edge; the definitions are self-explanatory.

We may drop the edge labelling of $\mathcal{G}$ to obtain a (multi)graph $\widetilde{\mathcal{G}}$ over the vertex set $\{0, \ldots, k\}$ : Each edge $\left\{\left(v_{1}, \lambda_{1}\right),\left(v_{2}, \lambda_{2}\right)\right\} \in E(\mathcal{G})$ gives rise to the edge $\left\{v_{1}, v_{2}\right\} \in E(\widetilde{G})$. We understand a path in $\mathcal{G}$ to be a sequence of edges in $E(\mathcal{G})$ such that two consecutive edges are adjacent in the graph $\widetilde{\mathcal{G}}$. This leads to the notions of connectedness of $\mathcal{G}$ and loops in $\mathcal{G}$.

The admissible graphs - i.e. graphs indexing a choice of pairings in the multiple commutator (6.7) - are generated by the following "growth process". We start with the empty graph $\mathcal{G}_{0}$, i.e. $E\left(\mathcal{G}_{0}\right)=\emptyset$. In a first step, we choose one or two empty edges of 1 of the same direction and connect each of them to an empty edge of 0 of opposite direction. Next, we choose one or two empty edges of 2 of the same direction and connect each of them to an empty edge of 0 or 1 of opposite direction. We continue in this manner for all vertices $3, \ldots, k$. We summarise some key properties of admissible graphs $\mathcal{G}$.

(a) $\mathcal{G}$ is connected.

(b) The degree of each $(v, \lambda)$ is either 0 or 1 .

(c) The labelled edge $\left\{\left(v_{1}, \lambda_{1}\right),\left(v_{2}, \lambda_{2}\right)\right\} \in E(\mathcal{G})$ only if $\lambda_{1}$ and $\lambda_{2}$ have opposite directions.

Property (c) implies that each graph $\mathcal{G}$ has a canonical directed representative, where each edge is ordered from the $a$-label to the $c$-label. See Fig. 6.1 for an example of such a graph.

We call a graph $\mathcal{G}$ of type $(p, k, l)$ whenever it is admissible and it contains $l$ loops. We denote by $\mathscr{G}(p, k, l)$ the set of graphs of type $(p, k, l)$. 


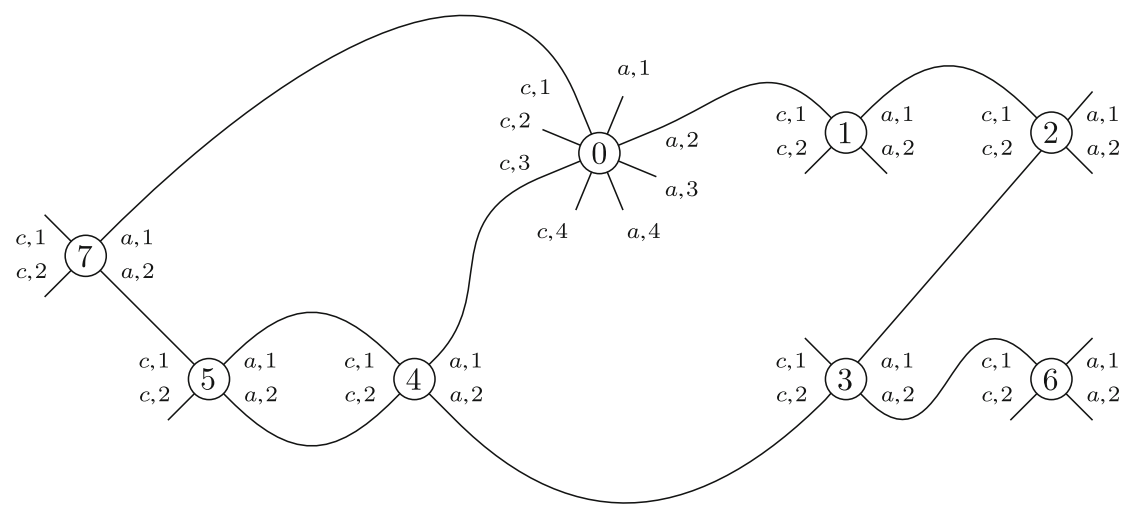

Fig. 6.1. An admissible graph of type $(p=4, k=7, l=3)$

By definition of admissible graphs, each contraction in (6.7) corresponds to a unique admissible graph. A contraction consists of at least $k$ and at most $2 k$ pairings. A contraction giving rise to a graph of type $(p, k, l)$ has $k+l$ pairings. The summand in (6.7) corresponding to any given $l$-loop contraction is given by an elementary term of the form

$$
\frac{(\mathrm{i} N)^{k}}{2^{k} N^{k+l}} \widehat{\mathrm{A}}_{N}\left(b^{(p+k-l)}\right),
$$

where the $(p+k-l)$-particle operator $b^{(p+k-l)}$ is of the form

$$
b^{(p+k-l)}=P_{ \pm} W_{i_{1} j_{1}, t_{v_{1}}} \cdots W_{i_{r} j_{r}, t_{v_{r}}}\left(a_{t}^{(p)} \otimes \mathbb{1}^{(k-l)}\right) W_{i_{r+1} j_{r+1}, t_{v_{r+1}}} \cdots W_{i_{k} j_{k}, t_{v_{k}}} P_{ \pm}
$$

for some $r=0, \ldots, k$. Indeed, the (anti)commutation relations (A.2) imply that each pairing produces a factor of $1 / N$. Furthermore, the creation and annihilation operators of each summand corresponding to any given contraction are (by definition) Wick ordered, and one readily sees that the associated integral kernel corresponds to an operator of the form (6.9). Thus we recover the splitting (4.4), whereby $G_{t, t_{1}, \ldots, t_{k}}^{(k, l)}\left(a^{(p)}\right)$ is a sum, indexed by all $l$-loop graphs, of elementary terms of the form (6.9).

As remarked above, we need to exploit the fact that many graphs have the same topological structure, i.e. can be identified after some permutation of the labels $\{1, \ldots, k\}$ of the vertices corresponding to interaction operators. We therefore define an equivalence relation on the set of graphs: $\mathcal{G} \sim \mathcal{G}^{\prime}$ if and only if there exists a permutation $\sigma \in S_{k}$ such that $\mathcal{G}^{\prime}=R_{\sigma}(\mathcal{G})$. Here $R_{\sigma}(\mathcal{G})$ is the graph defined by

$$
\left\{\left(v_{1}, \lambda_{1}\right),\left(v_{2}, \lambda_{2}\right)\right\} \in E\left(R_{\sigma}(\mathcal{G})\right) \Longleftrightarrow\left\{\left(\sigma\left(v_{1}\right), \lambda_{1}\right),\left(\sigma\left(v_{2}\right), \lambda_{2}\right)\right\} \in E(\mathcal{G}),
$$

where $\sigma(0) \equiv 0$. We call equivalence classes [G] graph structures, and denote the set of graph structures of admissible graphs of type $(p, k, l)$ by $\mathscr{Q}(p, k, l)$.

Note that, in general, $R_{\sigma}(\mathcal{G})$ need not be admissible if $\mathcal{G}$ is admissible. It is convenient to increase $\mathscr{G}(p, k, l)$ to include all $R_{\sigma}(\mathcal{G})$, where $\sigma \in S_{k}$ and $\mathcal{G}$ is admissible. In order to keep track of the admissible graphs in this larger set, we introduce the symbol $i_{\mathcal{G}}$ which is by definition 1 if $\mathcal{G} \in \mathscr{G}(p, k, l)$ is admissible and 0 otherwise. Because $R_{\sigma}(\mathcal{G}) \neq \mathcal{G}$ if $\sigma \neq \mathrm{id}$,

$$
|\mathscr{G}(p, k, l)|=k !|\mathscr{Q}(p, k, l)|
$$


Our goal is to find an upper bound on the number of graph structures of type $(p, k, l)$, which is sharp enough to show convergence of the Schwinger-Dyson series (4.2). Let us start with tree graphs: $l=0$. In this case the number of graph structures is equal to $2^{k}$ times the number of ordered trees ${ }^{1}$ with $k+1$ vertices, whose root has at most $2 p$ children and whose other vertices have at most 3 children. The factor $2^{k}$ arises from the fact that each vertex $v=1, \ldots, k$ can use either of the two empty edges of compatible direction to connect to its parent. We thus need some basic facts about ordered trees, which are covered in the following (more or less standard) combinatorial digression.

For $x, t \in \mathbb{R}$ and $n \in \mathbb{N}$ define

$$
A_{n}(x, t):=\frac{x}{x+n t}\left(\begin{array}{c}
x+n t \\
n
\end{array}\right)
$$

as well as $A_{0}(x, t):=1$. After some juggling with binomial coefficients one finds

$$
\sum_{k=0}^{n} A_{k}(x, t) A_{n-k}(y, t)=A_{n}(x+y, t) ;
$$

see [12] for details. Therefore

$$
\sum_{n_{1}+\cdots+n_{r}=n} A_{n_{1}}\left(x_{1}, t\right) \cdots A_{n_{r}}\left(x_{r}, t\right)=A_{n}\left(x_{1}+\cdots+x_{r}, t\right) .
$$

Set

$$
C_{n}^{m}:=A_{n}(1, m)=\frac{1}{1+n m}\left(\begin{array}{c}
1+n m \\
n
\end{array}\right)=\frac{1}{n(m-1)+1}\left(\begin{array}{c}
n m \\
n
\end{array}\right),
$$

the $n^{\text {th }} m$-ary Catalan number. Thus we have

$$
\sum_{n_{1}+\cdots+n_{r}=n} C_{n_{1}}^{m} \cdots C_{n_{r}}^{m}=\frac{r}{r+n m}\left(\begin{array}{c}
r+n m \\
n
\end{array}\right) .
$$

In particular,

$$
\sum_{n_{1}+\cdots+n_{m}=n-1} C_{n_{1}}^{m} \cdots C_{n_{m}}^{m}=C_{n}^{m} .
$$

Define an $m$-tree to be an ordered tree such that each vertex has at most $m$ children. The number of $m$-trees with $n$ vertices is equal to $C_{n}^{m}$. This follows immediately from $C_{0}^{m}=1$ and from (6.16), which expresses that all trees of order $n$ are obtained by adding $m$ (possibly empty) subtrees of combined order $n-1$ to the root.

We may now compute $|\mathscr{Q}(p, k, 0)|$. Since the root of the tree has at most $2 p$ children, we may express $|\mathscr{Q}(p, k, 0)|$ as the number of ordered forests comprising $2 p$ (possibly empty) 3 -trees whose combined order is equal to $k$. Therefore, by (6.15),

$$
|\mathscr{Q}(p, k, 0)|=2^{k} \sum_{n_{1}+\cdots+n_{2 p}=k} C_{n_{1}}^{3} \cdots C_{n_{2 p}}^{3}=2^{k} \frac{2 p}{2 p+3 k}\left(\begin{array}{c}
2 p+3 k \\
k
\end{array}\right) .
$$

Next, we extend this result to all values of $l$ in the form of an upper bound on $|\mathscr{Q}(p, k, l)|$.

\footnotetext{
1 An ordered tree is a rooted tree in which the children of each vertex are ordered.
} 
Lemma 6.2. Let $p, k, l \in \mathbb{N}$. Then

$$
|\mathscr{Q}(p, k, l)| \leq 2^{k}\left(\begin{array}{l}
k \\
l
\end{array}\right)\left(\begin{array}{c}
2 p+3 k \\
k
\end{array}\right)(p+k-l)^{l} .
$$

Proof. The idea is to remove edges from $\mathcal{G} \in \mathscr{G}(p, k, l)$ to obtain a tree graph, and then use the special case (6.17).

In addition to the properties (a) - (c) above, we need the following property of $\mathscr{G}(p, k, l)$ :

(d) If $\mathcal{G} \in \mathscr{G}(p, k, l)$ then there exists a subset $\mathcal{V} \subset\{1, \ldots, k\}$ of size $l$ and a choice of direction $\delta: \mathcal{V} \rightarrow\{a, c\}$ such that, for each $v \in \mathcal{V}$, both edges of $v$ with direction $\delta(v)$ are nonempty. Denote by $\mathcal{E}(v) \subset E(\mathcal{G})$ the set consisting of the two above edges. We additionally require that removing one of the two edges of $\mathcal{E}(v)$ from $\mathcal{G}$, for each $v \in \mathcal{V}$, yields a tree graph, with the property that, for each $v \in \mathcal{V}$, the remaining edge of $\mathcal{E}(v)$ is contained in the unique path connecting $v$ to the root.

This is an immediate consequence of the growth process for admissible graphs. The set $\mathcal{V}$ corresponds to the set of vertices whose addition produces two edges. Note that property (d) is independent of the representative and consequently holds also for non-admissible $\mathcal{G} \in \mathscr{G}(p, k, l)$.

Before coming to our main argument, we note that a tree graph $\mathcal{T} \in \mathscr{G}(p, k, 0)$ gives rise to a natural lexicographical order on the vertex set $\{1, \ldots, k\}$. Let $v \in\{1, \ldots, k\}$. There is a unique path that connects $v$ to the root. Denote by $0=v_{1}, v_{2}, \ldots, v_{q}=v$ the sequence of vertices along this path. For each $j=1, \ldots, q-1$, let $\lambda_{j}$ be the label of the edge $\left\{v_{j}, v_{j+1}\right\}$ at $v_{j}$. We assign to $v$ the string $S(v):=\left(\lambda_{1}, \ldots, \lambda_{q-1}\right)$. Choose some (fixed) ordering of the sets of labels $\{\lambda\}$, for each $v$. Then the set of vertices $\{1, \ldots, k\}$ is ordered according to the lexicographical order of the string $S(v)$.

We now start removing loops from a given graph $\mathcal{G} \in \mathscr{G}(p, k, l)$. Define $\mathcal{R}_{1}$ as the graph obtained from $\mathcal{G}$ by removing all edges in $\bigcup_{v \in \mathcal{V}} \mathcal{E}(v)$. By property (d) above, $\mathcal{R}_{1}$ is a forest comprising $l$ trees. Define $\mathcal{T}_{1}$ as the connected component of $\mathcal{R}_{1}$ containing the root. Now we claim that there is at least one $v \in \mathcal{V}$ such that both edges of $\mathcal{E}(v)$ are incident to a vertex of $\mathcal{T}_{1}$. Indeed, were this not the case, we could choose for each $v \in \mathcal{V}$ an edge in $\mathcal{E}(v)$ that is not incident to any vertex of $\mathcal{T}_{1}$. Call $\mathcal{R}_{1}^{\prime}$ the graph obtained by adding all such edges to $\mathcal{R}_{1}$. Now, since no vertex in $\mathcal{V}$ is in the connected component of $\mathcal{R}_{1}$, it follows that no vertex in $\mathcal{V}$ is in the connected component $\mathcal{R}_{1}^{\prime}$. This is a contradiction to property (d) which requires that $\mathcal{R}_{1}^{\prime}$ should be a (connected) tree.

Let us therefore consider the set $\tilde{\mathcal{V}}$ of all $v \in \mathcal{V}$ such that both edges of $\mathcal{E}(v)$ are incident to a vertex of $\mathcal{T}_{1}$. We have shown that $\tilde{\mathcal{V}} \neq \emptyset$. For each choice of $v$ and $e$, where $v \in \tilde{\mathcal{V}}$ and $e \in \mathcal{E}(v)$, we get a forest of $l-1$ trees by adding $e$ to the edge set of $\mathcal{R}_{1}$. Then $v$ is in the same tree as the root, so that each such choice of $v$ and $e$ yields a string $S(v)$ as described above. We choose $v_{1}$ and $e\left(v_{1}\right)$ as the unique couple that yields the smallest string (note that different choices have different strings). Finally, set $\mathcal{G}_{1}$ equal to $\mathcal{G}$ from which $e\left(v_{1}\right)$ has been removed, and $\mathcal{V}_{1}:=\mathcal{V} \backslash\{v\}$.

We have thus obtained an $(l-1)$-loop graph $\mathcal{G}_{1}$ and a set $\mathcal{V}_{1}$ of size $l-1$, which together satisfy the property $(\mathrm{d})$. We may therefore repeat the above procedure. In this manner we obtain the sequences $v_{1}, \ldots, v_{l}$ and $\mathcal{G}_{1}, \ldots, \mathcal{G}_{l}$. Note that $\mathcal{G}_{l}$ is obtained by removing the edges $e\left(v_{1}\right), \ldots, e\left(v_{l}\right)$ from $\mathcal{G}$, and is consequently a tree graph. Also, by construction, the sequence $v_{1}, \ldots, v_{l}$ is increasing in the lexicographical order of $\mathcal{G}_{l}$.

Next, consider the tree graph $\mathcal{G}_{l}$. Each edge $e\left(v_{j}\right)$ connects the single empty edge of $v_{j}$ with direction $\delta\left(v_{j}\right)$ with an empty edge of opposite direction of a vertex $v$, where 
$v$ is smaller than $v_{j}$ in the lexicographical order of $\mathcal{G}_{l}$. It is easy to see that, for each $j$, there are at most $(p+k-l)$ such connections.

We have thus shown that we can obtain any $\mathcal{G} \in \mathscr{G}(p, k, l)$ by choosing some tree $\mathcal{G}_{l} \in \mathscr{G}(p, k, 0)$, choosing $l$ elements $v_{j}$ out of $\{1, \ldots, k\}$, ordering them lexicographically (according to the order of $\left.\mathcal{G}_{l}\right)$ and choosing an edge out of at most $(p+k-l)$ possibilities for $v_{1}, \ldots, v_{l}$. Thus,

$$
|\mathscr{G}(p, k, l)| \leq\left(\begin{array}{l}
k \\
l
\end{array}\right)(p+k-l)^{l}|\mathscr{G}(p, k, 0)| .
$$

The claim then follows from (6.10) and (6.17).

6.3. Proof of convergence. We are now armed with everything we need in order to estimate $\int_{\Delta^{k}(t)} \mathrm{d} \underline{t} G_{t, \underline{t}}^{(k, l)}\left(a^{(p)}\right)$. Recall that

$$
G_{t, t_{1}, \ldots, t_{k}}^{(k, l)}\left(a^{(p)}\right)=\frac{\mathrm{i}^{k}}{2^{k}} \sum_{\mathcal{G} \in \mathscr{G}(p, k, l)} i_{\mathcal{G}} G_{t, t_{1}, \ldots, t_{k}}^{(k, l)(\mathcal{G})}\left(a^{(p)}\right),
$$

where $G_{t, t_{1}, \ldots, t_{k}}^{(k, l)(\mathcal{G})}\left(a^{(p)}\right)$ is an elementary term of the form (6.9) indexed by the graph $\mathcal{G}$. We rewrite this using graph structures. Pick some choice $\mathcal{P}: \mathscr{Q}(p, k, l) \rightarrow \mathscr{G}(p, k, l)$ of representatives. Then we get

$$
\begin{aligned}
G_{t, t_{1}, \ldots, t_{k}}^{(k, l)}\left(a^{(p)}\right) & =\frac{\mathrm{i}^{k}}{2^{k}} \sum_{\mathcal{Q} \in \mathcal{Q}(p, k, l)} \sum_{\mathcal{G} \in \mathcal{Q}} i_{\mathcal{G}} G_{t, t_{1}, \ldots, t_{k}}^{(k, l)(\mathcal{G})}\left(a^{(p)}\right) \\
& =\frac{\mathrm{i}^{k}}{2^{k}} \sum_{\mathcal{Q} \in \mathcal{Q}(p, k, l)} \sum_{\sigma \in S_{k}} i_{R_{\sigma}(\mathcal{P}(\mathcal{Q}))} G_{t, t_{1}, \ldots, t_{k}}^{(k, l)\left(R_{\sigma}(\mathcal{P}(\mathcal{Q}))\right)}\left(a^{(p)}\right) .
\end{aligned}
$$

Now, by definition of $R_{\sigma}$, we see that

$$
G_{t, t_{1}, \ldots, t_{k}}^{(k, l)\left(R_{\sigma}(\mathcal{G})\right)}\left(a^{(p)}\right)=G_{t, t_{\sigma(1)}, \ldots, t_{\sigma(k)}}^{(k, l)(\mathcal{G})}\left(a^{(p)}\right) .
$$

Thus,

$$
\begin{aligned}
\int_{\Delta^{k}(t)} \mathrm{d} \underline{t} G_{t, t_{1}, \ldots, t_{k}}^{(k, l)}\left(a^{(p)}\right) & =\frac{\mathrm{i}^{k}}{2^{k}} \sum_{\mathcal{Q} \in \mathcal{Q}(p, k, l)} \sum_{\sigma \in S_{k}} i_{R_{\sigma}(\mathcal{P}(\mathcal{Q}))} \int_{\Delta^{k}(t)} \mathrm{d} \underline{t}_{G_{t, t_{\sigma(1)}, \ldots, t_{\sigma(k)}}}\left(a^{(p)}\right) \\
& =\frac{\mathrm{i}^{k}}{2^{k}} \sum_{\mathcal{Q} \in \mathcal{Q}(p, k, l)} \int_{\Delta_{\mathcal{Q}^{k}(t)} \mathrm{d} \underline{t} G_{t, t_{1}, \ldots, t_{k}}^{(k, l)(\mathcal{P}(\mathcal{Q}))}\left(a^{(p)}\right),}
\end{aligned}
$$

where

$$
\Delta_{\mathcal{Q}}^{k}(t):=\left\{\left(t_{1}, \ldots, t_{k}\right): \exists \sigma \in S_{k}: i_{R_{\sigma}(\mathcal{P}(\mathcal{Q}))}=1,\left(t_{\sigma(1)}, \ldots, t_{\sigma(k)}\right) \in \Delta^{k}(t)\right\} \subset[0, t]^{k}
$$

is a union of disjoint simplices. 
Therefore, (6.5) and (6.9) imply, for any $\Phi^{(p+k-l)} \in \mathcal{H}_{ \pm}^{(p+k-l)}$, that

$$
\begin{aligned}
& \| \int_{\Delta^{k}(t)} \mathrm{d} \underline{t} G_{t, \underline{t}}^{(k, l)}\left(a^{(p)}\right) \Phi^{(p+k-l) \|} \\
& \leq \frac{1}{2^{k}} \sum_{\mathcal{Q} \in \mathscr{Q}(p, k, l)} \int_{\Delta_{\mathcal{Q}}^{k}(t)} \mathrm{d} \underline{t}\left\|G_{t, t_{1}, \ldots, t_{k}}^{(k, l)(\mathcal{Q}))}\left(a^{(p)}\right) \Phi^{(p+k-l)}\right\| \\
& \leq \frac{1}{2^{k}} \sum_{\mathcal{Q} \in \mathscr{Q}(p, k, l)} \int_{[0, t]^{k}} \mathrm{~d} \underline{t}\left\|G_{t, t_{1}, \ldots, t_{k}}^{(k, l)(\mathcal{Q}(\mathcal{Q}))}\left(a^{(p)}\right) \Phi^{(p+k-l)}\right\| \\
& \leq \frac{1}{2^{k}} \sum_{\mathcal{Q} \in \mathscr{Q}(p, k, l)}\left(\frac{\pi \kappa^{2} t}{2}\right)^{k / 2}\left\|a^{(p)}\right\|\left\|\Phi^{(p+k-l)}\right\| \\
& \leq\left(\begin{array}{c}
2 p+3 k \\
k
\end{array}\right)\left(\begin{array}{l}
k \\
l
\end{array}\right)(p+k-l)^{l}\left(\frac{\pi \kappa^{2} t}{2}\right)^{k / 2}\left\|a^{(p)}\right\|\left\|\Phi^{(p+k-l)}\right\|,
\end{aligned}
$$

where the last inequality follows from Lemma 6.2. Of course, the above treatment remains valid for regularised potentials. We summarise:

$$
\left\|G_{t}^{(k, l), \varepsilon}\left(a^{(p)}\right)\right\| \leq\left(\begin{array}{c}
2 p+3 k \\
k
\end{array}\right)\left(\begin{array}{l}
k \\
l
\end{array}\right)(p+k-l)^{l}\left(\frac{\pi \kappa^{2} t}{2}\right)^{k / 2}\left\|a^{(p)}\right\|,
$$

for all $\varepsilon \geq 0$.

Using (6.20) we may now proceed exactly as in the case of a bounded interaction potential. Let

$$
\rho(\kappa, v):=\frac{1}{128 \pi \kappa^{2} v^{2}} .
$$

The removal of the cutoff and summary of the results are contained in

Lemma 6.3. Let $t<\rho(\kappa, v)$. Then we have on $\mathcal{H}_{ \pm}^{(v N)}$

$$
\mathrm{e}^{\mathrm{i} t H_{N}} \widehat{\mathrm{A}}_{N}\left(a^{(p)}\right) \mathrm{e}^{-\mathrm{i} t H_{N}}=\sum_{k=0}^{\infty} \sum_{l=0}^{k} \frac{1}{N^{l}} \widehat{\mathrm{A}}_{N}\left(G_{t}^{(k, l)}\left(a^{(p)}\right)\right),
$$

in operator norm, uniformly in $N$. Furthermore, for $L \in \mathbb{N}$, we have the $1 / N$-expansion

$$
\mathrm{e}^{\mathrm{i} t H_{N}} \widehat{\mathrm{A}}_{N}\left(a^{(p)}\right) \mathrm{e}^{-\mathrm{i} t H_{N}}=\sum_{l=0}^{L-1} \frac{1}{N^{l}} \sum_{k=l}^{\infty} \widehat{\mathrm{A}}_{N}\left(G_{t}^{(k, l)}\left(a^{(p)}\right)\right)+O\left(\frac{1}{N^{L}}\right)
$$

where the sum converges on $\mathcal{H}_{ \pm}^{(v N)}$ uniformly in $N$.

Proof. Using (6.20) we may repeat the proof of Lemma 5.3 to the letter to prove the statements about convergence. Thus (6.22) holds for all $\varepsilon>0$. The proof of (6.22) for $\varepsilon=0$ follows by approximation and is banished to Appendix B.

\section{Mean-Field Limit}

In this section we identify the mean-field dynamics as the dynamics given by the Hartree equation. 
7.1. Hartree equation. The Hartree equation reads

$$
\mathrm{i} \partial_{t} \psi=h \psi+\left(w *|\psi|^{2}\right) \psi .
$$

It is the equation of motion of a classical Hamiltonian system with phase space $\Gamma:=$ $H^{1}\left(\mathbb{R}^{3}\right)$. Here $H^{1}\left(\mathbb{R}^{3}\right)$ is the usual Sobolev space of index one. In analogy to $\widehat{\mathrm{A}}_{N}$ we define $\mathrm{A}$ as the map from closed operators on $\mathcal{H}_{+}^{(p)}$ to functions on phase space, through

$$
\begin{aligned}
\mathrm{A}\left(a^{(p)}\right)(\psi): & \left\langle\psi^{\otimes p}, a^{(p)} \psi^{\otimes p}\right\rangle \\
= & \int \mathrm{d} x_{1} \cdots \mathrm{d} x_{p} \mathrm{~d} y_{1} \cdots \mathrm{d} y_{p} \bar{\psi}\left(x_{p}\right) \cdots \\
& \bar{\psi}\left(x_{1}\right) a^{(p)}\left(x_{1}, \ldots, x_{p} ; y_{1}, \ldots, y_{p}\right) \psi\left(y_{1}\right) \cdots \psi\left(y_{p}\right) .
\end{aligned}
$$

We define the space of "observables" $\mathfrak{A}$ as the linear hull of $\left\{A\left(a^{(p)}\right): p \in \mathbb{N}, a^{(p)} \in \mathcal{B}\right.$ $\left.\left(\mathcal{H}_{+}^{(p)}\right)\right\}$.

The Hamilton function is given by

$$
H:=\mathrm{A}(h)+\frac{1}{2} \mathrm{~A}(W),
$$

i.e.

$$
H(\psi)=\int \mathrm{d} x|\nabla \psi|^{2}+\frac{1}{2} \int \mathrm{d} x\left(w *|\psi|^{2}\right)|\psi|^{2}=\langle\psi, h \psi\rangle+\frac{1}{2}\left\langle\psi^{\otimes 2}, W \psi^{\otimes 2}\right\rangle .
$$

Using the Hardy-Littlewood-Sobolev and Sobolev inequalities (see e.g. [13]) one sees that $H(\psi)$ is well-defined on $\Gamma$ :

$$
\int \mathrm{d} x \mathrm{~d} y \frac{|\psi(x)|^{2}|\psi(y)|^{2}}{|x-y|} \lesssim\left\||\psi|^{2}\right\|_{6 / 5}^{2}=\|\psi\|_{12 / 5}^{4} \lesssim\|\psi\|_{H^{1}}^{4},
$$

where the symbol $\lesssim$ means the left side is bounded by the right side multiplied by a positive constant that is independent of $\psi$.

The Hartree equation is equivalent to

$$
\mathrm{i} \partial_{t} \psi=\partial_{\bar{\psi}} H(\psi) .
$$

The symplectic form on $\Gamma$ is given by

$$
\omega=\mathrm{i} \int \mathrm{d} x \mathrm{~d} \bar{\psi}(x) \wedge \mathrm{d} \psi(x),
$$

which induces a Poisson bracket given by

$$
\{\psi(x), \bar{\psi}(y)\}=\mathrm{i} \delta(x-y), \quad\{\psi(x), \psi(y)\}=\{\bar{\psi}(x), \bar{\psi}(y)\}=0 .
$$

For $A, B \in \mathfrak{A}$ we have that

$$
\{A, B\}(\psi)=\mathrm{i} \int \mathrm{d} x\left[\partial_{\psi} A(\psi) \partial_{\bar{\psi}} B(\psi)-\partial_{\psi} B(\psi) \partial_{\bar{\psi}} A(\psi)\right] .
$$

The "mass" function

$$
N(\psi):=\int \mathrm{d} x|\psi|^{2}
$$

is the generator of the gauge transformations $\psi \mapsto \mathrm{e}^{-\mathrm{i} \theta} \psi$. By the gauge invariance of the Hamiltonian, $\{H, N\}=0$, we conclude, at least formally, that $N$ is a conserved quantity. Similarly, the energy $H$ is formally conserved.

The space of observables $\mathfrak{A}$ has the following properties: 
(i) $\overline{\mathrm{A}\left(a^{(p)}\right)}=\mathrm{A}\left(\left(a^{(p)}\right)^{*}\right)$.

(ii) If $a^{(p)} \in \mathcal{B}\left(\mathcal{H}_{+}^{(p)}\right)$ and $b \in \mathcal{B}(\mathcal{H})$, then

$$
\mathrm{A}\left(a^{(p)}\right)(b \psi)=\mathrm{A}\left(\left(b^{*}\right)^{\otimes p} a^{(p)} b^{\otimes p}\right)(\psi) .
$$

(iii) If $a^{(p)}$ and $b^{(q)}$ are $p$ - and $q$-particle operators, respectively, then

$$
\left\{\mathrm{A}\left(a^{(p)}\right), \mathrm{A}\left(b^{(q)}\right)\right\}=\operatorname{ipq} \mathrm{A}\left(\left[a^{(p)}, b^{(q)}\right]_{1}\right) .
$$

(iv) If $a^{(p)} \in \mathcal{B}\left(\mathcal{H}_{+}^{(p)}\right)$, then

$$
\left\|\mathrm{A}\left(a^{(p)}\right)(\psi)\right\| \leq\left\|a^{(p)}\right\|\|\psi\|^{2 p} .
$$

The free time evolution

$$
\phi_{0}^{t}(\psi):=\mathrm{e}^{-\mathrm{i} t h} \psi
$$

is the Hamiltonian flow corresponding to the free Hamilton function $\mathrm{A}(h)$. We abbreviate the free time evolution of observables $A \in \mathfrak{A}$ by $A_{t}:=A \circ \phi_{0}^{t}$. Thus, $\mathrm{A}\left(a^{(p)}\right)_{t}=$ $\mathrm{A}\left(a_{t}^{(p)}\right)$.

In order to define the Hamiltonian flow on all of $L^{2}\left(\mathbb{R}^{3}\right)$, we rewrite the Hartree equation (7.1) with initial data $\psi(0)=\psi$ as an integral equation

$$
\psi(t)=\mathrm{e}^{-\mathrm{i} t h} \psi-\mathrm{i} \int_{0}^{t} \mathrm{~d} s \mathrm{e}^{-\mathrm{i}(t-s) h}\left(w *|\psi(s)|^{2}\right) \psi(s) .
$$

Lemma 7.1. Let $\psi \in L^{2}\left(\mathbb{R}^{3}\right)$. Then (7.5) has a unique global solution $\psi(\cdot) \in C$ $\left(\mathbb{R} ; L^{2}\left(\mathbb{R}^{3}\right)\right)$, which depends continuously on the initial data $\psi$. Furthermore, $\|\psi(t)\|=$ $\|\psi\|$ for all $t$. Finally, we have a Schwinger-Dyson expansion for observables: Let $a^{(p)} \in \mathcal{B}\left(\mathcal{H}_{+}^{(p)}\right), v>0$ and $t<\rho(\kappa, v)$. Then

$$
\begin{aligned}
\mathrm{A}\left(a^{(p)}\right)(\psi(t)) & =\sum_{k=0}^{\infty} \mathrm{A}\left(G_{t}^{(k, 0)}\left(a^{(p)}\right)\right)(\psi) \\
& =\sum_{k=0}^{\infty} \frac{1}{2^{k}} \int_{\Delta^{k}(t)} \mathrm{d} t\left\{\mathrm{~A}\left(W_{t_{k}}\right), \ldots\left\{\mathrm{A}\left(W_{t_{1}}\right), \mathrm{A}\left(a_{t}^{(p)}\right)\right\} \ldots\right\}(\psi),
\end{aligned}
$$

uniformly in the ball $B_{v}:=\left\{\psi \in L^{2}\left(\mathbb{R}^{3}\right):\|\psi\|^{2} \leq v\right\}$.

Proof. The well-posedness of (7.5) is a well-known result; see for instance [4,24]. The remaining statements follow from a "tree expansion", which also yields an existence result. We first use the Schwinger-Dyson expansion to construct an evolution on the space of observables. We then show that this evolution stems from a Hamiltonian flow that satisfies the Hartree equation (7.5).

First, we generalise our class of "observables" to functions that are not gauge invariant, i.e. that correspond to bounded operators $a^{(q, p)} \in \mathcal{B}\left(\mathcal{H}_{+}^{p} ; \mathcal{H}_{+}^{q}\right)$. We set $\mathrm{A}\left(a^{(q, p)}\right)$ $(\psi):=\left\langle\psi^{\otimes q}, a^{(q, p)} \psi^{\otimes p}\right\rangle$, and denote by $\widetilde{\mathfrak{A}}$ the linear hull of observables of the form $\mathrm{A}\left(a^{(q, p)}\right)$ with $a^{(q, p)} \in \mathcal{B}\left(\mathcal{H}_{+}^{p} ; \mathcal{H}_{+}^{q}\right)$. 
It is convenient to introduce the abbreviations

$$
G:=\{\mathrm{A}(h), \cdot\}, \quad D:=\frac{1}{2}\{\mathrm{~A}(W), \cdot\} .
$$

Then $\mathrm{e}^{G t}$ is well-defined on $\widetilde{\mathfrak{A}}$ through $\left(\mathrm{e}^{G t} A\right)(\psi)=A\left(\mathrm{e}^{-\mathrm{i} h} \psi\right)$, where $A \in \widetilde{\mathfrak{A}}$. Note also that

$$
D_{s}:=\mathrm{e}^{G s} D \mathrm{e}^{-G s}=\frac{1}{2}\left\{\mathrm{~A}\left(W_{s}\right), \cdot\right\} .
$$

Let $A \in \widetilde{\mathfrak{A}}$. We use the Schwinger-Dyson series for $\mathrm{e}^{(G+D) t}$ to define the flow $S(t) A$ through

$$
\begin{aligned}
S(t) A & :=\sum_{k=0}^{\infty} \int_{\Delta^{k}(t)} \mathrm{d} \underline{t} D_{t_{k}} \cdots D_{t_{1}} \mathrm{e}^{G t} A \\
& \left.=\sum_{k=0}^{\infty} \int_{\Delta^{k}(t)} \mathrm{d} \underline{t} \frac{1}{2^{k}}\left\{\mathrm{~A}\left(W_{t_{k}}\right), \ldots\left\{\mathrm{A}\left(W_{t_{1}}\right), A_{t}\right)\right\} \ldots\right\} .
\end{aligned}
$$

Our first task is to show convergence of (7.7) for small times.

Let $A=\mathrm{A}\left(a^{(q, p)}\right)$. As with (7.3) one finds, after short computation, that

$$
\frac{1}{2}\left\{\mathrm{~A}(W), \mathrm{A}\left(a^{(q, p)}\right)\right\}=\mathrm{A}\left(\mathrm{i} \sum_{i=1}^{q} W_{i q+1}\left(a^{(q, p)} \otimes \mathbb{1}\right)-\mathrm{i} \sum_{i=1}^{p}\left(a^{(q, p)} \otimes \mathbb{1}\right) W_{i p+1}\right)
$$

Thus we see that the nested Poisson brackets in (7.7) yield a "tree expansion" which may be described as follows. Define $T_{t, t_{1}, \ldots, t_{k}}^{(k)}\left(a^{(q, p)}\right)$ recursively through

$$
\begin{aligned}
T_{t}^{(0)}\left(a^{(q, p)}\right):= & a_{t}^{(q, p)} \\
T_{t, t_{1}, \ldots, t_{k}}^{(k)}\left(a^{(q, p)}\right):= & \mathrm{i} P_{+} \sum_{i=1}^{q+k-1} W_{i q+k, t_{k}}\left(T_{t, t_{1}, \ldots, t_{k-1}}^{(k-1)}\left(a^{(q, p)}\right) \otimes \mathbb{1}\right) P_{+} \\
& -\mathrm{i} P_{+} \sum_{i=1}^{p+k-1}\left(T_{t, t_{1}, \ldots, t_{k-1}}^{(k-1)}\left(a^{(q, p)}\right) \otimes \mathbb{1}\right) W_{i p+k, t_{k}} P_{+} .
\end{aligned}
$$

Note that $T_{t, t_{1}, \ldots, t_{k}}^{(k)}\left(a^{(q, p)}\right)$ is an operator from $\mathcal{H}_{+}^{(p+k)}$ to $\mathcal{H}_{+}^{(q+k)}$. Moreover, (7.8) implies that

$$
\frac{1}{2^{k}}\left\{\mathrm{~A}\left(W_{t_{k}}\right), \ldots\left\{\mathrm{A}\left(W_{t_{1}}\right), \mathrm{A}\left(a_{t}^{(q, p)}\right)\right\} \ldots\right\}=\mathrm{A}\left(T_{t, t_{1}, \ldots, t_{k}}^{(k)}\left(a^{(q, p)}\right)\right)
$$

Also, by definition, we see that for gauge-invariant observables $a^{(p)}$ we have

$$
T_{t, t_{1}, \ldots, t_{k}}^{(k)}\left(a^{(p)}\right)=G_{t, t_{1}, \ldots, t_{k}}^{(k, 0)}\left(a^{(p)}\right) .
$$

We may use the methods of Sect. 6 to obtain the desired estimate. One sees that $T_{t, t_{1}, \ldots, t_{k}}^{(k)}\left(a^{(p)}\right)$ is a sum of elementary terms, indexed by labelled ordered trees, whose 
root has degree at most $p+q$, and whose other vertices have at most 3 children. From (6.15) we find that there are

$$
\frac{p+q}{p+q+3 k}\left(\begin{array}{c}
p+q+3 k \\
k
\end{array}\right)
$$

unlabelled trees of this kind. Proceeding exactly as in Sect. 6 we find that

$\int_{\Delta^{k}(t)} \mathrm{d} \underline{t}\left\|T_{t, t_{1}, \ldots, t_{k}}^{(k)}\left(a^{(q, p)}\right) \Phi^{(p+k)}\right\| \leq\left(\begin{array}{c}p+q+3 k \\ k\end{array}\right)\left(\frac{\pi \kappa^{2} t}{2}\right)^{k / 2}\left\|a^{(q, p)}\right\|\left\|\Phi^{(p+k)}\right\|$,

where $\Phi^{(p+k)} \in \mathcal{H}_{+}^{(p+k)}$. Let $\psi \in L^{2}\left(\mathbb{R}^{3}\right)$ with $\|\psi\|^{2} \leq v$. Then $\left|\mathrm{A}\left(a^{(q, p)}\right)(\psi)\right| \leq$ $\left\|a^{(q, p)}\right\|\|\psi\|^{p+q}$ implies

$$
\begin{aligned}
& \int_{\Delta^{k}(t)} \mathrm{d} t\left|\frac{1}{2^{k}}\left\{\mathrm{~A}\left(W_{t_{k}}\right), \ldots\left\{\mathrm{A}\left(W_{t_{1}}\right), \mathrm{A}\left(a_{t}^{(q, p)}\right)\right\} \ldots\right\}(\psi)\right| \\
& \leq\left(\begin{array}{c}
p+q+3 k \\
k
\end{array}\right)\left(\frac{\pi \kappa^{2} t}{2}\right)^{k / 2}\left\|a^{(q, p)}\right\| v^{k+(p+q) / 2} .
\end{aligned}
$$

Convergence of the Schwinger-Dyson series (7.7) for small times $t$ follows immediately.

Thus, for small times $t$, the flow $S(t)$ is well-defined on $\widetilde{\mathfrak{A}}$, and it is easy to check that it satisfies the equation

$$
S(t) A=\mathrm{e}^{G t} A+\int_{0}^{t} \mathrm{~d} s S(s) D \mathrm{e}^{G(t-s)} A,
$$

for all $A \in \widetilde{\mathfrak{A}}$.

In order to establish a link with the Hartree equation (7.5), we consider $f \in L^{2}\left(\mathbb{R}^{3}\right)$ and define the function $F_{f} \in \widetilde{\mathfrak{A}}$ through $F_{f}(\psi):=\langle f, \psi\rangle$. Clearly, the mapping $f \mapsto\left(S(t) F_{f}\right)(\psi)$ is antilinear and (7.10) implies that it is bounded. Thus there exists a unique vector $\psi(t)$ such that

$$
\left(S(t) F_{f}\right)(\psi)=:\langle f, \psi(t)\rangle .
$$

We now proceed to show that $(S(t) A)(\psi)=A(\psi(t))$ for all $A \in \widetilde{\mathfrak{A}}$. By definition, this is true for $A=F_{f}$. As a first step, we show that

$$
S(t)(A B)=(S(t) A)(S(t) B)
$$

where $A, B \in \widehat{\mathfrak{A}}$. Write

$$
\begin{aligned}
S(t)(A B) & =\sum_{k=0}^{\infty} \int_{\Delta^{k}(t)} \mathrm{d} \underline{t} D_{t_{k}} \cdots D_{t_{1}} \mathrm{e}^{G t}(A B) \\
& =\sum_{k=0}^{\infty} \int_{\Delta^{k}(t)} \mathrm{d} \underline{t} D_{t_{k}} \cdots D_{t_{1}}\left(A_{t} B_{t}\right),
\end{aligned}
$$

where we used $\mathrm{e}^{G t}(A B)=\left(\mathrm{e}^{G t} A\right)\left(\mathrm{e}^{G t} B\right)$. We now claim that

$$
\int_{\Delta^{k}(t)} \mathrm{d} \underline{t} D_{t_{k}} \cdots D_{t_{1}}\left(A_{t} B_{t}\right)=\sum_{l+m=k} \int_{\Delta^{l}(t)} \mathrm{d} \underline{t} \int_{\Delta^{m}(t)} \mathrm{d} \underline{s}\left(D_{t_{l}} \cdots D_{t_{1}} A_{t}\right)\left(D_{s_{m}} \cdots D_{s_{1}} B_{t}\right),
$$


where the sum ranges over $l, m \geq 0$. This follows easily by induction on $k$ and using $D_{s}(A B)=A\left(D_{s} B\right)+\left(D_{s} A\right) B$. Then (7.12) follows immediately.

Next, we note that (7.12) implies that $(S(t) A)(\psi)=A(\psi(t))$, whenever $A$ is of the form $A=\mathrm{A}\left(a^{(q, p)}\right)$, where

$$
a^{(q, p)}=\sum_{j} P_{+}\left|f_{1}^{j} \otimes \cdots \otimes f_{q}^{j}\right\rangle\left\langle g_{1}^{j} \otimes \cdots \otimes g_{p}^{j}\right| P_{+},
$$

where the sum is finite, and $f_{i}^{j}, g_{i}^{j} \in L^{2}\left(\mathbb{R}^{3}\right)$. Now each $a^{(q, p)} \in \mathcal{B}\left(\mathcal{H}_{+}^{(p)} ; \mathcal{H}_{+}^{(q)}\right)$ can be written as the weak operator limit of a sequence $\left(a_{n}^{(q, p)}\right)_{n \in \mathbb{N}}$ of operators of type (7.14). One sees immediately that

$$
\lim _{n} \mathrm{~A}\left(a_{n}^{(q, p)}\right)(\psi(t))=\mathrm{A}\left(a^{(q, p)}\right)(\psi(t)) .
$$

On the other hand, uniform boundedness implies that $\sup _{n}\left\|a_{n}^{(q, p)}\right\|<\infty$, so that

$$
\begin{gathered}
\left\langle\psi^{\otimes(q+k)}, W_{i_{1} j_{1}, t_{v_{1}}} \cdots W_{i_{r} j_{r}, t_{v_{r}}}\left(a_{n}^{(q, p)} \otimes \mathbb{1}^{(k)}\right) W_{i_{r+1} j_{r+1}, t_{v_{r+1}}} \cdots W_{i_{k} j_{k}, t_{v_{k}}} \psi^{\otimes(p+k)}\right\rangle \\
\leq\left\|a_{n}^{(q, p)}\right\|\left\|W_{i_{r} j_{r}, t_{v_{r}}} \cdots W_{i_{1} j_{1}, t_{v_{1}}} \psi^{\otimes(q+k)}\right\|\left\|W_{i_{r+1} j_{r+1}, t_{v_{r+1}}} \cdots W_{i_{k} j_{k}, t_{v_{k}}} \psi^{\otimes(p+k)}\right\|
\end{gathered}
$$

justifies the use of dominated convergence in

$$
\lim _{n}\left(S(t) \mathrm{A}\left(a_{n}^{(q, p)}\right)\right)(\psi)=\left(S(t) \mathrm{A}\left(a^{(q, p)}\right)\right)(\psi) .
$$

We have thus shown that

$$
(S(t) A)(\psi)=A(\psi(t)), \quad \forall A \in \widetilde{\mathfrak{A}} .
$$

Let us now return to (7.11). Setting $A=F_{f}$, we find that (7.11) implies

$$
\begin{aligned}
\langle f, \psi(t)\rangle & =\left\langle f, \mathrm{e}^{-i h} \psi\right\rangle+\int_{0}^{t} \mathrm{~d} s \frac{1}{2}\left(S(s)\left\{\mathrm{A}(W),\left(F_{f}\right)_{t-s}\right\}\right)(\psi) \\
& =\left\langle f, \mathrm{e}^{-i h} \psi\right\rangle+\int_{0}^{t} \mathrm{~d} s\left(\left\{\mathrm{~A}(W),\left(F_{f}\right)_{t-s}\right\}\right)(\psi(s)),
\end{aligned}
$$

where we used (7.15). Using (7.8) we thus find

$$
\langle f, \psi(t)\rangle=\left\langle f, \mathrm{e}^{-i h} \psi\right\rangle-\mathrm{i} \int_{0}^{t} \mathrm{~d} s\left\langle\left(\mathrm{e}^{\mathrm{i} h(t-s)} f\right) \otimes \psi(s), W \psi(s) \otimes \psi(s)\right\rangle,
$$

which is exactly the Hartree equation (7.5) projected onto $f$. We have thus shown that $\psi(t)$ as defined above solves the Hartree equation.

To show norm-conservation we abbreviate $F(s):=\left(w *|\psi(s)|^{2}\right) \psi(s)$ and write, using (7.5),

$$
\begin{aligned}
\|\psi(t)\|^{2}-\|\psi\|^{2}= & \mathrm{i} \int_{0}^{t} \mathrm{~d} s\left[\left\langle F(s), \mathrm{e}^{-\mathrm{i} s h} \psi\right\rangle-\left\langle\mathrm{e}^{-\mathrm{i} s h} \psi, F(s)\right\rangle\right] \\
& +\int_{0}^{t} \mathrm{~d} s \int_{0}^{t} \mathrm{~d} r\left\langle\mathrm{e}^{\mathrm{i} s h} F(s), \mathrm{e}^{\mathrm{i} r h} F(r)\right\rangle .
\end{aligned}
$$


The last term is equal to

$$
\int_{0}^{t} \mathrm{~d} s \int_{0}^{s} \mathrm{~d} r\left[\left\langle\mathrm{e}^{\mathrm{i} s h} F(s), \mathrm{e}^{\mathrm{i} r h} F(r)\right\rangle+\left\langle\mathrm{e}^{\mathrm{i} r h} F(r), \mathrm{e}^{\mathrm{i} s h} F(s)\right\rangle\right] .
$$

Therefore (7.5) implies that

$$
\|\psi(t)\|^{2}-\|\psi\|^{2}=\mathrm{i} \int_{0}^{t} \mathrm{~d} s\langle F(s), \psi(s)\rangle-\mathrm{i} \int_{0}^{t} \mathrm{~d} s\langle\psi(s), F(s)\rangle=0,
$$

since $\langle F(s), \psi(s)\rangle \in \mathbb{R}$, as can be seen by explicit calculation. Thus we can iterate the above existence result for short times to obtain a global solution.

Furthermore, (7.16) implies that $\psi(t)$ is weakly continuous in $t$. Since the norm of $\psi(t)$ is conserved, $\psi(t)$ is strongly continuous in $t$. Similarly, the Schwinger-Dyson expansion (7.7) implies that the map $\psi \mapsto \psi(t)$ is weakly continuous for small times, uniformly in $\|\psi\|$ in compacts. Therefore, the map $\psi \mapsto \psi(t)$ is weakly continuous for all times $t$, and norm-conservation implies that it is strongly continuous.

7.2. Wick quantisation. In order to state our main result in a general setting, we shortly discuss how the many-body quantum mechanics of bosons can be viewed as a deformation quantisation of the (classical) Hartree theory. The deformation parameter (the analogue of $\hbar$ in the usual quantisation of classical theories) is $1 / N$. We define quantisation as the linear map $\widehat{(\cdot)_{N}}: \mathfrak{A} \rightarrow \widehat{\mathfrak{A}}$ defined by the formal replacement $\psi(x) \mapsto \widehat{\psi}_{N}(x)$ and $\bar{\psi}(x) \mapsto \widehat{\psi}_{N}^{*}(x)$ followed by Wick ordering. In other words,

$$
\widehat{(\cdot)}_{N}: \mathrm{A}\left(a^{(p)}\right) \mapsto \widehat{\mathrm{A}}_{N}\left(a^{(p)}\right) .
$$

Extending the definition of $\widehat{(\cdot)_{N}}$ to unbounded operators in the obvious way, we see that $\widehat{H}_{N}$ is the quantisation of $H$.

Note that (3.3) and (7.3) imply, for $A, B \in \mathfrak{A}$,

$$
\left.\left[\widehat{A}_{N}, \widehat{B}_{N}\right]=\frac{N^{-1}}{\mathrm{i}} \widehat{\{A, B}\right\}_{N}+O\left(\frac{1}{N^{2}}\right),
$$

so that $1 / N$ is indeed the deformation parameter of $\widehat{(\cdot)}_{N}$.

7.3. Mean-field limit: A Egorov-type result. Let $\phi^{t}$ denote the Hamiltonian flow of the Hartree equation on $L^{2}\left(\mathbb{R}^{3}\right)$. Introduce the short-hand notation

$$
\begin{array}{rlrl}
\alpha^{t} A & :=A \circ \phi^{t}, & & A \in \mathfrak{A}, \\
\widehat{\alpha}^{t} \mathbf{A} & :=\mathrm{e}^{\mathrm{i} t N \widehat{H}_{N}} \mathbf{A} \mathrm{e}^{-\mathrm{i} t N \widehat{H}_{N}}, & \mathbf{A} \in \widehat{\mathfrak{A}} .
\end{array}
$$

We may now state and prove our main result, which essentially says that, in the mean-field limit $n=v N \rightarrow \infty$, time evolution and quantisation commute.

Theorem 7.2. Let $A \in \mathfrak{A}, v>0$, and $\varepsilon>0$. Then there exists a function $A(t) \in \mathfrak{A}$ such that

$$
\sup _{t \in \mathbb{R}}\left\|\alpha^{t} A-A(t)\right\|_{L^{\infty}\left(B_{v}\right)} \leq \varepsilon
$$

as well as

$$
\left\|\left.\left(\widehat{\alpha}^{t} \widehat{A}_{N}-\widehat{A(t)}_{N}\right)\right|_{\mathcal{H}_{+}^{(v N)}}\right\| \leq \varepsilon+\frac{C(\varepsilon, v, t, A)}{N} .
$$


Remark. The "intermediate function" $A(t)$ is required, since the full time evolution $\alpha^{t}$ does not leave $\mathfrak{A}$ invariant.

Proof. Most of the work has already been done in the previous sections. Without loss of generality take $A=\mathrm{A}\left(a^{(p)}\right)$ for some $p \in \mathbb{N}$ and $a^{(p)} \in \mathcal{B}\left(\mathcal{H}_{ \pm}^{(p)}\right)$. Assume that $t<\rho(\kappa, v)$. Taking $L=1$ in (6.23) we get

$$
\left.\widehat{\alpha}^{t} \widehat{\mathrm{A}}_{N}\left(a^{(p)}\right)\right|_{\mathcal{H}_{+}^{(v N)}}=\left.\sum_{k=0}^{\infty} \widehat{\mathrm{A}}_{N}\left(G_{t}^{(k, 0)}\left(a^{(p)}\right)\right)\right|_{\mathcal{H}_{+}^{(v N)}}+O\left(\frac{1}{N}\right) .
$$

Comparing this with (7.6) immediately yields

$$
\widehat{\alpha}^{t} \widehat{\mathrm{A}}_{N}\left(a^{(p)}\right)=\left[\alpha^{t} \mathrm{~A}\left(a^{(p)}\right) \widehat{]_{N}}+O\left(\frac{1}{N}\right)\right.
$$

on $\mathcal{H}_{+}^{(v N)}$, where $\left[\alpha^{t} \mathrm{~A}\left(a^{(p)}\right)\right]_{N}$ is defined through its norm-convergent power series. This is the statement of the theorem for short times.

The extension to all times follows from an iteration argument. We postpone the details to the proof of Theorem 7.3 below. In its notation $A(t)$ is given by

$$
A(t)=\sum_{k_{1}=0}^{K_{1}-1} \cdots \sum_{k_{m}=0}^{K_{m}-1} \mathrm{~A}\left(G_{\tau}^{\left(k_{m}, 0\right)} G_{\tau}^{\left(k_{m-1}, 0\right)} \cdots G_{\tau}^{\left(k_{1}, 0\right)} a^{(p)}\right) .
$$

The result may also be expressed in terms of coherent states.

Theorem 7.3. Let $a^{(p)} \in \mathcal{B}\left(\mathcal{H}_{+}^{(p)}\right), \psi \in L^{2}\left(\mathbb{R}^{3}\right)$ with $\|\psi\|=1$, and $T>0$. Then there exist constants $C, \beta>0$, depending only on $p$, $T$ and $\kappa$, such that

$$
\begin{aligned}
& \left|\left\langle\psi^{\otimes N}, \mathrm{e}^{\mathrm{i} t H_{N}} \widehat{\mathrm{A}}_{N}\left(a^{(p)}\right) \mathrm{e}^{-\mathrm{i} t H_{N}} \psi^{\otimes N}\right\rangle-\left\langle\psi(t)^{\otimes p}, a^{(p)} \psi(t)^{\otimes p}\right\rangle\right| \\
& \quad \leq \frac{C}{N^{\beta}}\left\|a^{(p)}\right\|, \quad t \in[0, T] .
\end{aligned}
$$

Here $\psi(t)$ is the solution to the Hartree equation (7.5) with initial data $\psi$.

Proof. Introduce a cutoff $K \in \mathbb{N}$ and write (in self-explanatory notation)

$$
\begin{aligned}
\widehat{\alpha}^{\tau} \widehat{\mathrm{A}}_{N}\left(a^{(p)}\right) & =\sum_{k=0}^{K-1} \widehat{\mathrm{A}}_{N}\left(G_{\tau}^{(k, 0)}\left(a^{(p)}\right)\right)+\widehat{\alpha}_{\geq K}^{\tau} \widehat{\mathrm{A}}_{N}\left(a^{(p)}\right)+\frac{1}{N} R_{N, \tau}\left(a^{(p)}\right), \\
\alpha^{\tau} \mathrm{A}\left(a^{(p)}\right) & =\sum_{k=0}^{K-1} \mathrm{~A}\left(G_{\tau}^{(k, 0)}\left(a^{(p)}\right)\right)+\alpha_{\geq K}^{\tau} \mathrm{A}\left(a^{(p)}\right) .
\end{aligned}
$$


To avoid cluttering the notation, from now on we drop the parentheses of the linear map $G_{\tau}^{(k, 0)}$. We iterate (7.19) $m$ times by applying it to its first term and get

$$
\begin{aligned}
& \left(\widehat{\alpha}^{\tau}\right)^{m} \widehat{\mathrm{A}}_{N}\left(a^{(p)}\right) \\
& =\sum_{k_{1}=0}^{K_{1}-1} \cdots \sum_{k_{m}=0}^{K_{m}-1} \widehat{\mathrm{A}}_{N}\left(G_{\tau}^{\left(k_{m}, 0\right)} G_{\tau}^{\left(k_{m-1}, 0\right)} \cdots G_{\tau}^{\left(k_{1}, 0\right)} a^{(p)}\right)+\left(\widehat{\alpha}^{\tau}\right)^{m-1} \widehat{\alpha}_{\geq K_{1}}^{\tau} \widehat{\mathrm{A}}_{N}\left(a^{(p)}\right) \\
& \quad+\sum_{j=1}^{m-1} \sum_{k_{1}=0}^{K_{1}-1} \cdots \sum_{k_{j}=0}^{K_{j}-1}\left(\widehat{\alpha}^{\tau}\right)^{m-1-j} \widehat{\alpha}_{\geq K_{j+1}} \widehat{\mathrm{A}}_{N}\left(G_{\tau}^{\left(k_{j}, 0\right)} G_{\tau}^{\left(k_{j-1}, 0\right)} \cdots G_{\tau}^{\left(k_{1}, 0\right)} a^{(p)}\right) \\
& +\frac{1}{N}\left(\widehat{\alpha}^{\tau}\right)^{m-1} R_{N, \tau}\left(a^{(p)}\right) \\
& \quad+\frac{1}{N} \sum_{j=1}^{m-1} \sum_{k_{1}=0}^{K_{1}-1} \ldots \sum_{k_{j}=0}^{K_{j}-1}\left(\widehat{\alpha}^{\tau}\right)^{m-1-j} R_{N, \tau}\left(G_{\tau}^{\left(k_{j}, 0\right)} \cdots G_{\tau}^{\left(k_{1}, 0\right)} a^{(p)}\right) .
\end{aligned}
$$

A similar expression without the third line holds for $\left(\alpha^{\tau}\right)^{m} \mathrm{~A}\left(a^{(p)}\right)$.

In order to control this somewhat unpleasant expression, we abbreviate

$$
x:=\sqrt{\frac{\tau}{\rho(\kappa, 1)}} .
$$

Assume that $x<1$. Then (6.20) and (6.23) imply the estimates, valid on $\mathcal{H}_{+}^{(N)}$,

$$
\begin{aligned}
\left\|G_{\tau}^{(k, 0)} a^{(p)}\right\| & \leq 4^{p}\left\|a^{(p)}\right\| x^{k}, \\
\left\|\widehat{\alpha}_{\geq K}^{\tau} \widehat{\mathrm{A}}_{N}\left(a^{(p)}\right)\right\| & \leq 4^{p}\left\|a^{(p)}\right\| \frac{x^{K}}{1-x}, \\
\left\|R_{N, \tau}\left(a^{(p)}\right)\right\| & \leq(4 \mathrm{e})^{p}\left\|a^{(p)}\right\| \frac{x}{(1-x)^{3}} .
\end{aligned}
$$

Furthermore, (7.6) implies that

$$
\left\|\alpha_{\geq K}^{\tau} \mathrm{A}\left(a^{(p)}\right)\right\|_{L^{\infty}\left(B_{1}\right)} \leq 4^{p}\left\|a^{(p)}\right\| \frac{x^{K}}{1-x} .
$$

We also need

$$
\begin{aligned}
\left|\left\langle\psi^{\otimes N}, \widehat{\mathrm{A}}_{N}\left(a^{(p)}\right) \psi^{\otimes N}\right\rangle-\mathrm{A}\left(a^{(p)}\right)(\psi)\right| & =\left|\frac{N \cdots(N-p+1)}{N^{p}}-1\right|\left|\mathrm{A}\left(a^{(p)}\right)(\psi)\right| \\
& \leq \sum_{j=1}^{p-1}\left|\frac{N \cdots(N-j)}{N^{j+1}}-\frac{N \cdots(N-j+1)}{N^{j}}\right|\left\|a^{(p)}\right\| \\
& \leq \frac{p^{2}}{N}\left\|a^{(p)}\right\| .
\end{aligned}
$$


Armed with these estimates we may now complete the proof of Theorem 7.3. Suppose that $1 / 2 \leq x<1$. Then

$$
\begin{aligned}
& \sum_{k_{1}=0}^{K_{1}-1} \cdots \sum_{k_{m}=0}^{K_{m}-1} \mid\left\langle\psi^{\otimes N}, \widehat{\mathrm{A}}_{N}\left(G_{\tau}^{\left(k_{m}, 0\right)} G_{\tau}^{\left(k_{m-1}, 0\right)} \cdots G_{\tau}^{\left(k_{1}, 0\right)} a^{(p)}\right) \psi \psi^{\otimes N}\right\rangle \\
& \quad-\mathrm{A}\left(G_{\tau}^{\left(k_{m}, 0\right)} G_{\tau}^{\left(k_{m-1}, 0\right)} \cdots G_{\tau}^{\left(k_{1}, 0\right)} a^{(p)}\right)(\psi) \mid \\
& \quad \leq \frac{1}{N}\left(p+K_{1}+\cdots+K_{m}\right)^{2} 4^{m\left(p+K_{1}+\cdots+K_{m}\right)}\left\|a^{(p)}\right\| .
\end{aligned}
$$

Similarly, the second line of (7.21) on $\mathcal{H}_{+}^{(N)}$ and its classical equivalent on $B_{1}$ are bounded by

$$
\sum_{j=1}^{m} x^{K_{j}} 4^{j\left(p+K_{1}+\cdots+K_{j-1}\right)}\left\|a^{(p)}\right\| .
$$

Finally, the last line of (7.21) on $\mathcal{H}_{+}^{(N)}$ is bounded by

$$
\frac{1}{N} \sum_{j=1}^{m} 4^{(j+1)\left(p+K_{1}+\cdots+K_{j-1}\right)}\left\|a^{(p)}\right\| .
$$

Now pick $m$ large enough that $T \leq m \tau$. Then it is easy to check that there exist $a_{1}, \ldots, a_{m}$ such that setting

$$
K_{j}=a_{j} \log N, \quad j=1, \ldots, m
$$

implies that the three above expressions are all bounded by $C N^{-\beta}\left\|a^{(p)}\right\|$, for some $\beta>0$. This remains of course true for all $m^{\prime} \leq m$. Since any time $t \leq T$ can be reached by at most $m$ iterations with $1 / 2 \leq x<1$, the claim follows.

We conclude with a short discussion on density matrices. First we recall some standard results; see for instance [18]. Let $\Gamma \in \mathcal{L}^{1}$, where $\mathcal{L}^{1}$ is the space of trace class operators on some Hilbert space. Equipped with the norm $\|\Gamma\|_{1}:=\operatorname{Tr}|\Gamma|, \mathcal{L}^{1}$ is a Banach space. Its dual is equal to $\mathcal{B}$, the space of bounded operators, and the dual pairing is given by

$$
\langle A, \Gamma\rangle=\operatorname{Tr}(A \Gamma), \quad A \in \mathcal{B}, \Gamma \in \mathcal{L}^{1} .
$$

Therefore,

$$
\|\Gamma\|_{1}=\sup _{A \in \mathcal{B},\|A\| \leq 1}|\operatorname{Tr}(A \Gamma)|
$$

Consider an $N$-particle density matrix $0 \leq \Gamma_{N} \in \mathcal{L}^{1}\left(\mathcal{H}_{+}^{(N)}\right)$ that satisfies $\operatorname{Tr} \Gamma_{N}=1$ and is symmetric in the sense that $\Gamma_{N} P_{+}=\bar{\Gamma}_{N}$. Define the $p$-particle marginals

$$
\Gamma_{N}^{(p)}:=\operatorname{Tr}_{p+1, \ldots, N} \Gamma_{N},
$$

where $\operatorname{Tr}_{p+1, \ldots, N}$ denotes the partial trace over the coordinates $p+1, \ldots, N$. Define furthermore

$$
\Gamma_{N}(t)=\mathrm{e}^{-\mathrm{i} t H_{N}} \Gamma_{N} \mathrm{e}^{\mathrm{i} t H_{N}},
$$


as well as the $p$-particle marginals $\Gamma_{N}^{(p)}(t)$ of $\Gamma_{N}(t)$.

Noting that

$$
\operatorname{Tr}\left(\widehat{\mathrm{A}}_{N}\left(a^{(p)}\right) \Gamma_{N}(t)\right)=\frac{p !}{N^{p}}\left(\begin{array}{c}
N \\
p
\end{array}\right) \operatorname{Tr}\left(a^{(p)} \Gamma_{N}^{(p)}(t)\right)=\operatorname{Tr}\left(a^{(p)} \Gamma_{N}^{(p)}(t)\right)+O\left(\frac{1}{N}\right),
$$

we see that (7.23) and Theorem 7.3 imply

Corollary 7.4. Let $\psi \in \mathcal{H}$ with $\|\psi\|=1$, and let $\psi(t)$ be the solution of (7.5) with initial data $\psi$. Set $\Gamma_{N}:=(|\psi\rangle\langle\psi|)^{\otimes N}$. Then, for any $p \in \mathbb{N}$ and $T>0$ there exist constants $C, \beta>0$, depending only on $p, T$ and $\kappa$, such that

$$
\left\|\Gamma_{N}^{(p)}(t)-(\psi(t)\rangle\langle\psi(t))^{\otimes p}\right\|_{1} \leq \frac{C}{N^{\beta}}, \quad t \in[0, T] .
$$

Remark. Actually it is enough for $\Gamma_{N}$ to factorise asymptotically. If $\left(\Gamma_{N}\right)_{N \in \mathbb{N}}$ is a sequence of symmetric density matrices satisfying

$$
\lim _{N \rightarrow \infty} \| \Gamma_{N}^{(1)}-|\psi\rangle\langle\psi| \|_{1}=0
$$

then one finds

$$
\lim _{N \rightarrow 0} \| \Gamma_{N}^{(1)}(t)-|\psi(t)\rangle\langle\psi(t)| \|_{1}=0, \quad t \in \mathbb{R} .
$$

This is a straightforward corollary of the proof of Theorem 7.3. By an argument of Lieb and Seiringer (see the remark after Theorem 1 in [14]), this implies that

$$
\lim _{N \rightarrow 0}\left\|\Gamma_{N}^{(p)}(t)-(|\psi(t)\rangle\langle\psi(t)|)^{\otimes p}\right\|_{1}=0, \quad t \in \mathbb{R}
$$

for all $p$.

\section{Some Generalisations}

In this section we generalise our results to a larger class of interaction potentials, and allow an external potential. For this we need Strichartz estimates for Lorentz spaces. We start with a short summary of the relevant results (see [1,11]).

For $1 \leq q \leq \infty$ and $0<\theta<1$ we define the real interpolation functor $(\cdot, \cdot)_{\theta, q}$ as follows. Let $A_{0}$ and $A_{1}$ be two Banach spaces contained in some larger Banach space $A$. Define the real interpolation norm

$$
\|a\|_{\left(A_{0}, A_{1}\right)_{\theta, q}}:= \begin{cases}{\left[\int_{0}^{\infty}\left(t^{-\theta} K(t, a)\right)^{q} \mathrm{~d} t / t\right]^{1 / q},} & q<\infty \\ \sup _{t \geq 0} t^{-\theta} K(t, a), & q=\infty\end{cases}
$$

where

$$
K(t, a):=\inf _{a=a_{0}+a_{1}}\left(\left\|a_{0}\right\|_{A_{0}}+t\left\|a_{1}\right\|_{A_{1}}\right) .
$$

Define $\left(A_{0}, A_{1}\right)_{\theta, q}$ as the space of $a \in A$ such that $\|a\|_{\left(A_{0}, A_{1}\right)_{\theta, q}}<\infty$. Then $\left(A_{0}, A_{1}\right)_{\theta, q}$ is a Banach space. The Lorentz space $L^{p, q}\left(\mathbb{R}^{3}, \mathrm{~d} x\right) \equiv L^{p, q}$ is defined by interpolation as

$$
L^{p, q}:=\left(L^{p_{0}}, L^{p_{1}}\right)_{\theta, q},
$$


where $1 \leq p_{0}, p_{1} \leq \infty, p_{0} \neq p_{1}$, and

$$
\frac{1}{p}=\frac{1-\theta}{p_{0}}+\frac{\theta}{p_{1}} .
$$

Lorentz spaces have the following properties that are of interest to us. First, $L^{p, p}=$ $L^{p}$. Second, $L^{p, \infty}=L_{w}^{p}$, where $L_{w}^{p}$ is the weak $L^{p}$ space (see e.g. [1,19]). In particular, we have for the Coulomb potential in 3 dimensions

$$
\frac{1}{|x|} \in L^{3, \infty}
$$

Finally, Lorentz spaces satisfy a general Hölder inequality (see [17]): Let $1<p, p_{1}, p_{2}<$ $\infty$ and $1 \leq q, q_{1}, q_{2} \leq \infty$ satisfy

$$
\frac{1}{p_{1}}+\frac{1}{p_{2}}=\frac{1}{p}, \quad \frac{1}{q_{1}}+\frac{1}{q_{2}}=\frac{1}{q} .
$$

Then we have

$$
\|f g\|_{L^{p, q}} \lesssim\|f\|_{L^{p_{1}, q_{1}}}\|g\|_{L^{p_{2}, q_{2}}} .
$$

We need an endpoint homogeneous Strichartz estimate proved in [11]. For a map $f: \mathbb{R} \rightarrow L^{p, q}$ we define the space-time norm

$$
\|f\|_{L_{t}^{r} L_{x}^{p, q}}:=\left[\int \mathrm{d} t\|f(t)\|_{L^{p, q}}^{r}\right]^{1 / r} .
$$

Then Theorem 10.1 of [11] implies that

$$
\left\|\mathrm{e}^{\mathrm{i} t \Delta} f\right\|_{L_{t}^{r} L_{x}^{p, 2}} \lesssim\|f\|_{L^{2}}
$$

whenever $2 \leq r<\infty$ and

$$
\frac{2}{r}+\frac{3}{p}=\frac{3}{2}
$$

We are now set for proving a generalisation of (6.2).

Lemma 8.1. Let $w \in L_{w}^{3}+L^{\infty}$. Then there is a constant $C=C(w)>0$, such that

$$
\int_{0}^{1}\left\|w \mathrm{e}^{\mathrm{i} t \Delta} \psi\right\|^{2} \mathrm{~d} t \leq C\|\psi\|^{2} .
$$

Proof. Let $w=w_{1}+w_{2}$ with $w_{1} \in L^{\infty}$ and $w_{2} \in L_{w}^{3}$. Then

$$
\left\|w \mathrm{e}^{\mathrm{i} t \Delta} \psi\right\|_{L_{t}^{2} L_{x}^{2}} \leq\left\|w_{1} \mathrm{e}^{\mathrm{i} t \Delta} \psi\right\|_{L_{t}^{2} L_{x}^{2}}+\left\|w_{2} \mathrm{e}^{\mathrm{i} t \Delta} \psi\right\|_{L_{t}^{2} L_{x}^{2}} .
$$

The first term is bounded by $\left\|w_{1}\right\|_{L^{\infty}}\|\psi\|_{L^{2}}$. To bound the second we use (8.1) and (8.2) with $r=2$ and $p=6$ to get

$$
\left\|w_{2} \mathrm{e}^{\mathrm{i} t \Delta} \psi\right\|_{L_{t}^{2} L_{x}^{2}} \lesssim\left\|w_{2}\right\|_{L^{3, \infty}}\left\|\mathrm{e}^{\mathrm{i} t \Delta} \psi\right\|_{L_{t}^{2} L_{x}^{6,2}} \lesssim\left\|w_{2}\right\|_{L^{3, \infty}}\|\psi\|_{L^{2}}
$$

Therefore,

$$
\left\|w \mathrm{e}^{\mathrm{i} t \Delta} \psi\right\|_{L_{t}^{2} L_{x}^{2}} \leq \sqrt{C(w)}\|\psi\|_{L^{2}}
$$


Now let us assume that $v, w \in L^{\infty}+L_{w}^{3}$. Set $\left.H_{0}\right|_{\mathcal{H}_{ \pm}^{(n)}}:=\sum_{i=1}^{n}-\Delta_{i}$. Then the required generalisation of Lemma 6.1 is

Lemma 8.2. There exists a constant $C \equiv C(w, v)$ such that

$$
\begin{gathered}
\int_{0}^{1}\left\|W_{i j} \mathrm{e}^{-\mathrm{i} t H_{0}} \Phi^{(n)}\right\|^{2} \mathrm{~d} t \leq C\left\|\Phi^{(n)}\right\|^{2}, \\
\int_{0}^{1}\left\|V_{i} \mathrm{e}^{-\mathrm{i} t H_{0}} \Phi^{(n)}\right\|^{2} \mathrm{~d} t \leq C\left\|\Phi^{(n)}\right\|^{2},
\end{gathered}
$$

where $\Phi^{(n)} \in \mathcal{H}_{ \pm}^{(n)}$.

Proof. The claim for $V$ follows immediately from Lemma 8.1. The estimate for $W$ follows similarly by using centre of mass coordinates.

Finally, we briefly discuss the changes to the combinatorics arising from an external potential. We classify the elementary terms according to the numbers $(k, l, m)$, where $k$ is the order of the multiple commutator, $l$ is the number of loops, and $m$ is the number of $V$-operators. Thus, instead of (4.5), we have the recursive definition

$$
\begin{aligned}
& G_{t, t_{1}, \ldots, t_{k}}^{(k, l, m)}\left(a^{(p)}\right)=\mathrm{i}(p+k-l-m-1)\left[W_{t_{k}}, G_{t, t_{1}, \ldots, t_{k-1}}^{(k-1, l, m)}\left(a^{(p)}\right)\right]_{1} \\
& +\mathrm{i}\left(\begin{array}{c}
p+k-l-m \\
2
\end{array}\right)\left[W_{t_{k}}, G_{t, t_{1}, \ldots, t_{k-1}}^{(k-1, l-1, m)}\left(a^{(p)}\right)\right]_{2} \\
& +\mathrm{i}(p+k-l-m)\left[V_{t_{k}}, G_{t, t_{1}, \ldots, t_{k-1}}^{(k-1, l, m-1)}\left(a^{(p)}\right)\right]_{1} \\
& p+k-l-m-1 \\
& =\mathrm{i} P_{ \pm} \sum_{i=1}^{p+k-l-m-1}\left[W_{i p+k-l-m, t_{k}}, G_{t, t_{1}, \ldots, t_{k-1}}^{(k-1, l, m)}\left(a^{(p)}\right) \otimes \mathbb{1}\right] P_{ \pm} \\
& +\mathrm{i} P_{ \pm} \sum_{1 \leq i<j \leq p+k-l-m}\left[W_{i j, t_{k}}, G_{t, t_{1}, \ldots, t_{k-1}}^{(k-1, l-1, m)}\left(a^{(p)}\right)\right] P_{ \pm} \\
& +\mathrm{i} P_{ \pm} \sum_{i=1}^{p+k-l-m}\left[V_{i, t_{k}}, G_{t, t_{1}, \ldots, t_{k-1}}^{(k-1, l, m-1)}\left(a^{(p)}\right)\right] P_{ \pm}
\end{aligned}
$$

as well as $G_{t}^{(0,0,0)}\left(a^{(p)}\right):=a_{t}^{(p)}$. We also set $G_{t, t_{1}, \ldots, t_{k}}^{(k, l, m)}\left(a^{(p)}\right)=0$ unless $0 \leq l \leq k-m$. It is again an easy exercise to show by induction on $k$ that

$$
\frac{(\mathrm{i} N)^{k}}{2^{k}}\left[\widehat{\mathrm{A}}_{N}\left(W_{t_{k}}\right), \ldots\left[\widehat{\mathrm{A}}_{N}\left(W_{t_{1}}\right), \widehat{\mathrm{A}}_{N}\left(a_{t}^{(p)}\right)\right] \ldots\right]=\sum_{l=0}^{k} \sum_{m=0}^{k-l} \frac{1}{N^{l}} \widehat{\mathrm{A}}_{N}\left(G_{t, t_{1}, \ldots, t_{k}}^{(k, l, m)}\left(a^{(p)}\right)\right) .
$$

Note that $G_{t, t_{1}, \ldots, t_{k}}^{(k, l, m)}\left(a^{(p)}\right)$ is a $p+k-l-m$ particle operator.

The graphs of Sect. 6 have to be modified: Each vertex corresponding to a $V$-operator has one edge for each direction $d=a, c$ (see Fig. 8.1).

Let us first consider tree graphs, $l=0$. Take the set of trees without an external potential as in Sect. 6. By allowing each vertex $v=1, \ldots, k$ whose edges $(a, 2)$ and $(c, 2)$ are empty to stand for either an interaction potential $W$ or an external potential $V$, we count all trees with an external potential. Thus, for a given $m$, there are at most 


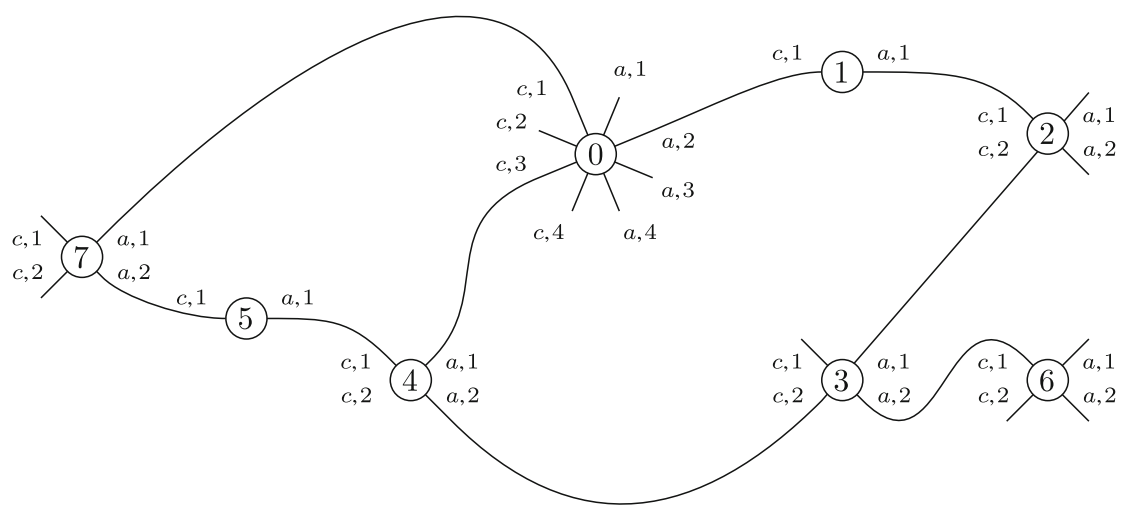

Fig. 8.1. An admissible graph of type $(p=4, k=7, l=2, m=2)$

$\left(\begin{array}{c}k \\ m\end{array}\right)|\mathscr{G}(p, k, 0)|$ tree graphs contributing to $G_{t, t_{1}, \ldots, t_{k}}^{(k, 0, m)}\left(a^{(p)}\right)$. If $l>0$ we repeat the argument in the proof Lemma 6.2, and find that the number of graph structures contributing to $G_{t, t_{1}, \ldots, t_{k}}^{(k, l, m)}\left(a^{(p)}\right)$ is bounded by

$$
2^{k}\left(\begin{array}{l}
k \\
m
\end{array}\right)\left(\begin{array}{l}
k \\
l
\end{array}\right)\left(\begin{array}{c}
2 p+3 k \\
k
\end{array}\right)(p+k-l-m)^{l} .
$$

Putting all this together, we find that

$$
\left\|G_{t}^{(k, l, m)}\left(a^{(p)}\right)\right\| \leq\left(\begin{array}{l}
k \\
m
\end{array}\right)\left(\begin{array}{l}
k \\
l
\end{array}\right)\left(\begin{array}{c}
2 p+3 k \\
k
\end{array}\right)(p+k-l-m)^{l}(C t)^{k / 2}\left\|a^{(p)}\right\| .
$$

Using the condition $p+k-l-m \leq n$, it is then easy to see that all convergence estimates remain valid with the additional factor $2^{k}$.

In summary, all of the results of Sects. 6 and 7 hold if

$$
v, w \in L_{w}^{3}+L^{\infty}
$$

\section{A. Second Quantisation}

We briefly summarise the main ingredients of many-body quantum mechanics and second quantisation. See for instance [2] for an extensive discussion.

Let $\mathcal{H}=L^{2}\left(\mathbb{R}^{d}, \mathrm{~d} x\right)$ be the "one-particle Hilbert space", where $d \in \mathbb{N}$. Manybody quantum mechanics is formulated on subspaces of the $n$-particle spaces $\mathcal{H}^{\otimes n}$. Let $P_{ \pm}^{(n)} \equiv P_{ \pm}$be the orthogonal projector onto the symmetric/antisymmetric subspace of $\mathcal{H}^{\otimes n}$, i.e.

$$
\left(P_{ \pm} \Phi^{(n)}\right)\left(x_{1}, \ldots, x_{n}\right):=\frac{1}{n !} \sum_{\sigma \in S_{n}}( \pm 1)^{|\sigma|} \Phi^{(n)}\left(x_{\sigma(1)}, \ldots, x_{\sigma(n)}\right),
$$

where $|\sigma|$ denotes the number of transpositions in the permutation $\sigma$, and $\Phi^{(n)} \in \mathcal{H}^{\otimes n}$. We define the bosonic $n$-particle space as $\mathcal{H}_{+}^{(n)}:=P_{+} \mathcal{H}^{\otimes n}$, and the fermionic $n$-particle space as $\mathcal{H}_{-}^{(n)}:=P_{-} \mathcal{H}^{\otimes n}$. We adopt the usual convention that $\mathcal{H}^{\otimes 0}=\mathbb{C}$. 
We introduce the Fock space

$$
\mathcal{F}_{ \pm}(\mathcal{H}) \equiv \mathcal{F}_{ \pm}:=\bigoplus_{n=0}^{\infty} \mathcal{H}_{ \pm}^{(n)}
$$

A state $\Phi \in \mathcal{F}_{ \pm}$is a sequence $\Phi=\left(\Phi^{(n)}\right)_{n=0}^{\infty}$, where $\Phi^{(n)} \in \mathcal{H}_{ \pm}^{(n)}$. Equipped with the scalar product

$$
\langle\Phi, \Psi\rangle=\sum_{n=0}^{\infty}\left\langle\Phi^{(n)}, \Psi^{(n)}\right\rangle
$$

$\mathcal{F}_{ \pm}$is a Hilbert space. The vector $\Omega:=(1,0,0, \ldots)$ is called the vacuum. By a slight abuse of notation, we denote a vector of the form $\Phi=\left(0, \ldots, 0, \Phi^{(n)}, 0, \ldots\right) \in \mathcal{F}_{ \pm}$by its non-vanishing $n$-particle component $\Phi^{(n)}$. Define also the subspace of vectors with a finite particle number

$$
\mathcal{F}_{ \pm}^{0}:=\left\{\Phi \in \mathcal{F}_{ \pm}: \Phi^{(n)}=0 \text { for all but finitely many } n\right\} .
$$

On $\mathcal{F}_{ \pm}$we have the usual creation and annihilation operators, $\widehat{\psi}^{*}$ and $\widehat{\psi}$, which map the one-particle space $\mathcal{H}$ into densely defined closable operators on $\mathcal{F}_{ \pm}$. For $f \in \mathcal{H}$ and $\Phi \in \mathcal{F}_{ \pm}$, they are defined by

$$
\begin{aligned}
\left(\widehat{\psi}^{*}(f) \Phi\right)^{(n)}\left(x_{1}, \ldots, x_{n}\right) & :=\frac{1}{\sqrt{n}} \sum_{i=1}^{n}( \pm 1)^{i-1} f\left(x_{i}\right) \Phi^{(n-1)}\left(x_{1}, \ldots, x_{i-1}, x_{i+1}, \ldots, x_{n}\right), \\
\widehat{\psi}(f) \Phi)^{(n)}\left(x_{1}, \ldots, x_{n}\right) & :=\sqrt{n+1} \int \mathrm{d} y \bar{f}(y) \Phi^{(n+1)}\left(y, x_{1}, \ldots, x_{n}\right) .
\end{aligned}
$$

It is not hard to see that $\widehat{\psi}(f)$ and $\widehat{\psi}^{*}(f)$ are adjoints of each other (see for instance [2] for details). Furthermore, they satisfy the canonical (anti)commutation relations

$$
\left[\widehat{\psi}(f), \widehat{\psi}^{*}(g)\right]_{\mp}=\langle f, g\rangle \mathbb{1}, \quad\left[\widehat{\psi}^{\#}(f), \widehat{\psi}^{\#}(g)\right]_{\mp}=0,
$$

where $[A, B]_{\mp}:=A B \mp B A$, and $\widehat{\psi}^{\#}=\widehat{\psi}^{*}$ or $\widehat{\psi}$. In order to simplify notation, we usually identify $c \mathbb{1}$ with $c$, where $c \in \mathbb{C}$.

For our purposes, it is more natural to work with the rescaled creation and annihilation operators

$$
\widehat{\psi}_{N}^{\#}:=\frac{1}{\sqrt{N}} \widehat{\psi}^{\#},
$$

where $N>0$. We also introduce the operator-valued distributions defined formally by

$$
\widehat{\psi}_{N}^{\#}(x):=\widehat{\psi}_{N}^{\#}\left(\delta_{x}\right),
$$

where $\delta_{x}$ is the delta function at $x$. The formal expression $\widehat{\psi}_{N}^{\#}(x)$ has a rigorous meaning as a densely defined sesquilinear form on $\mathcal{F}_{ \pm}$(see [19] for details). In particular one has that

$$
\widehat{\psi}_{N}(f)=\int \mathrm{d} x \bar{f}(x) \widehat{\psi}_{N}(x), \quad \widehat{\psi}_{N}^{*}(f)=\int \mathrm{d} x f(x) \widehat{\psi}_{N}^{*}(x) .
$$

Furthermore, the (anti)commutation relations (A.1) imply that

$$
\left[\widehat{\psi}_{N}(x), \widehat{\psi}_{N}^{*}(y)\right]_{\mp}=\frac{1}{N} \delta(x-y), \quad\left[\widehat{\psi}_{N}^{\#}(x), \widehat{\psi}_{N}^{\#}(y)\right]_{\mp}=0
$$




\section{B. The Limit $\varepsilon \rightarrow 0$ in Lemma 6.3}

What remains is the justification of the equality in (6.22) for $\varepsilon=0$. Our strategy is to show that both sides of (6.23) with $\varepsilon>0$ converge strongly to the same expression with $\varepsilon=0$.

We first show the strong convergence of $G_{t}^{(k, l), \varepsilon}\left(a^{(p)}\right)$. Let $\Phi^{(n)} \in \mathscr{H}_{ \pm}^{(n)}$ and consider

$$
\left\|\left(W_{i j, s}^{\varepsilon}-W_{i j, s}\right) \Phi^{(n)}\right\|=\left\|I_{\left\{\left|W_{i j}\right|>\varepsilon^{-1}\right\}} W_{i j} e^{-i s H_{0}} \Phi^{(n)}\right\| \leq\left\|W_{i j} e^{-i s H_{0}} \Phi^{(n)}\right\| .
$$

Since the right side is in $L^{1}([0, t])$, we may use dominated convergence to conclude that

$$
\lim _{\varepsilon \rightarrow 0} \int_{0}^{t} \mathrm{~d} s\left\|\left(W_{i j, s}^{\varepsilon}-W_{i j, s}\right) \Phi^{(n)}\right\|=0 .
$$

Now

$$
\begin{aligned}
& \int_{0}^{t} \mathrm{~d} s \int_{0}^{t} \mathrm{~d} s^{\prime}\left\|W_{i j, s}^{\varepsilon} W_{i^{\prime} j^{\prime}, s^{\prime}}^{\varepsilon} \Phi^{(n)}-W_{i j, s} W_{i^{\prime} j^{\prime}, s^{\prime}} \Phi^{(n)}\right\| \\
& \leq \int_{0}^{t} \mathrm{~d} s \int_{0}^{t} \mathrm{~d} s^{\prime}\left\|W_{i j, s}^{\varepsilon} W_{i^{\prime} j^{\prime}, s^{\prime}}^{\varepsilon} \Phi^{(n)}-W_{i j, s}^{\varepsilon} W_{i^{\prime} j^{\prime}, s^{\prime}} \Phi^{(n)}\right\| \\
& \quad+\int_{0}^{t} \mathrm{~d} s \int_{0}^{t} \mathrm{~d} s^{\prime}\left\|W_{i j, s}^{\varepsilon} W_{i^{\prime} j^{\prime}, s^{\prime}} \Phi^{(n)}-W_{i j, s} W_{i^{\prime} j^{\prime}, s^{\prime}} \Phi^{(n)}\right\| .
\end{aligned}
$$

The first term is bounded by

$$
\left(\frac{\pi \kappa^{2} t}{2}\right)^{1 / 2} \int_{0}^{t} \mathrm{~d} s^{\prime}\left\|W_{i^{\prime} j^{\prime}, s^{\prime}}^{\varepsilon} \Phi^{(n)}-W_{i^{\prime} j^{\prime}, s^{\prime}} \Phi^{(n)}\right\| \rightarrow 0, \quad \varepsilon \rightarrow 0 .
$$

The integrand of the second term is bounded by $2\left\|W_{i j, s} W_{i^{\prime} j^{\prime}, s^{\prime}} \Phi^{(n)}\right\| \in L^{1}\left([0, t]^{2}\right)$, so that dominated convergence implies that the second term vanishes in the limit $\varepsilon \rightarrow 0$. A straightforward generalisation of this argument shows that

$$
G_{t}^{(k, l), \varepsilon}\left(a^{(p)}\right) \Phi^{(p+k-l)} \rightarrow G_{t}^{(k, l)}\left(a^{(p)}\right) \Phi^{(p+k-l)},
$$

as claimed. Since the series (6.22) converges uniformly in $\varepsilon$, we find that

$$
\sum_{k=0}^{\infty} \sum_{l=0}^{k} \frac{1}{N^{l}} \widehat{\mathrm{A}}_{N}\left(G_{t}^{(k, l), \varepsilon}\left(a^{(p)}\right)\right) \Phi^{(n)} \rightarrow \sum_{k=0}^{\infty} \sum_{l=0}^{k} \frac{1}{N^{l}} \widehat{\mathrm{A}}_{N}\left(G_{t}^{(k, l)}\left(a^{(p)}\right)\right) \Phi^{(n)},
$$

as $\varepsilon \rightarrow 0$.

Next, we show that $\mathrm{e}^{-\mathrm{i} t H_{N}^{\varepsilon} \Phi^{(n)}} \rightarrow \mathrm{e}^{-\mathrm{i} t H_{N}} \Phi^{(n)}$. This follows from strong resolvent convergence of $H_{N}^{\varepsilon}$ to $H_{N}$ as $\varepsilon \rightarrow 0$ by Trotter's theorem [18]. Let $W^{\varepsilon}:=\sum_{i<j} W_{i j}^{\varepsilon}$, and consider

$$
\begin{aligned}
N\left\|\left(H_{N}^{\varepsilon}-\mathrm{i}\right)^{-1} \Phi^{(n)}-\left(H_{N}-\mathrm{i}\right)^{-1} \Phi^{(n)}\right\| & =\left\|\left(H_{N}^{\varepsilon}-\mathrm{i}\right)^{-1}\left(W-W^{\varepsilon}\right)\left(H_{N}-\mathrm{i}\right)^{-1} \Phi^{(n)}\right\| \\
& \leq\left\|\left(W-W^{\varepsilon}\right)\left(H_{N}-\mathrm{i}\right)^{-1} \Phi^{(n)}\right\| .
\end{aligned}
$$

Clearly $\Psi^{(n)}:=\left(H_{N}-\mathrm{i}\right)^{-1} \Phi^{(n)}$ is in the domain of $H_{N}$. By the Kato-Rellich theorem [19], $\Psi^{(n)}$ is in the domain of $W_{i j}$ for all $i, j$. Therefore,

$$
\left\|\left(W_{i j}-W_{i j}^{\varepsilon}\right)\left(H_{N}-\mathrm{i}\right)^{-1} \Phi^{(n)}\right\|=\left\|I_{\left\{\left|W_{i j}\right|>\varepsilon^{-1}\right\}} W_{i j} \Psi^{(n)}\right\| \rightarrow 0
$$


as $\varepsilon \rightarrow 0$. Therefore

$$
\mathrm{e}^{\mathrm{i} t H_{N}^{\varepsilon}} \widehat{A}_{N}\left(a^{(p)}\right) \mathrm{e}^{-\mathrm{i} t H_{N}^{\varepsilon}} \Phi^{(n)} \rightarrow \mathrm{e}^{\mathrm{i} t H_{N}} \widehat{A}_{N}\left(a^{(p)}\right) \mathrm{e}^{-\mathrm{i} t H_{N}} \Phi^{(n)}
$$

as $\varepsilon \rightarrow 0$, and the proof is complete.

Acknowledgements. We thank W. De Roeck, S. Graffi and A. Pizzo for useful discussions and encouragement. We should also like to thank a referee for pointing out Ref. [14] in connection with the remark following Corollary 7.4.

\section{References}

1. Bergh, J., Löfström, J.: Interpolation Spaces, an Introduction. Berlin-Heidelberg-New York: Springer, 1976

2. Bratteli, O., Robinson, D.W.: Operator Algebras and Quantum Statistical Mechanics 2. BerlinHeidelberg-New York: Springer, 2002

3. Brown, W., Hepp, K.: The Vlasov dynamics and its fluctuations in the $1 / N$ limit of interacting classical particles. Commun. Math. Phys. 56, 101-113 (1977)

4. Chadam, J.M., Glassey, R.T.: Global existence of solutions to the Cauchy problem for time-dependent Hartree equations. J. Math. Phys. 16, 1122 (1975)

5. Egorov, Y.V.: The canonical transformations of pseudodifferential operators. Usp. Mat. Nauk 25, 235-236 (1969)

6. Erdős, L., Yau, H.-T.: Derivation of the nonlinear Schrödinger equation with Coulomb potential. Adv. Theor. Math. Phys. 5, 1169-1205 (2001)

7. Fröhlich, J., Graffi, S., Schwarz, S.: Mean-field and classical limit of many-body Schrödinger dynamics for bosons. Commun. Math. Phys. 271, 681-697 (2007)

8. Fröhlich, J., Knowles, A., Pizzo, A.: Atomism and Quantization. J. Phys. A 40, 3033-3045 (2007)

9. Ginibre, J., Velo, G.: The classical field limit of scattering theory for non-relativistic many-boson systems. I-II. Commun. Math. Phys. 66, 37-76 (1979); Commun. Math. Phys. 68, 45-68 (1979)

10. Hepp, K.: The classical limit for quantum mechanical correlation functions. Commun. Math. Phys. 35, 265-277 (1974)

11. Keel, M., Tao, T.: Endpoint Strichartz estimates. Amer. J. Math. 120, 955-980 (1998)

12. Knuth, D.E.: The Art of Computer Programming, Vol. 1, Reading, MA: Addison-Wesley, 1998

13. Lieb, E.H., Loss, M.: Analysis. Providence, RI: Amer. Math. Soc., 2001

14. Lieb, E.H., Seiringer, R.: Proof of Bose-Einstein condensation for dilute trapped gases. Phys. Rev. Lett. 88(17), 170409 (2002)

15. Narnhofer, H., Sewell, G.L.: Vlasov hydrodynamics of a quantum mechanical model. Commun. Math. Phys. 79, 9-24 (1981)

16. Neunzert, H.: Fluid Dyn. Trans. 9, 229 (1977); Neunzert, H.: Neuere qualitative und numerische Methoden in der Plasmaphysik. Paderborn: Vorlesungsmanuskript, 1975

17. O’Neil, R.: Convolution operators and $L(p, q)$ spaces. Duke Math. J. 30, 129-142 (1963)

18. Reed, M., Simon, B.: Methods of Modern Mathematical Physics I: Functional Analysis. New York: Academic Press, 1980

19. Reed, M., Simon, B.: Methods of Modern Mathematical Physics II: Fourier Analysis, Self-Adjointness. New York: Academic Press, 1975

20. Reed, M., Simon, B.: Methods of Modern Mathematical Physics IV: Analysis of Operators. New York: Academic Press, 1978

21. Rodnianski, I., Schlein, B.: Quantum fluctuations and rate of convergence towards mean field dynamics. http://arXiv.org/abs/0711.3087v1[math-ph], 2007

22. Schlein, B., Erdős, L.: Quantum Dynamics with Mean Field Interactions: a New Approach. http://arXiv. org/abs/0804.3774v1 (2008)

23. Simon, B.: Best constants in some operator smoothness estimates. J. Func. Anal. 107, 66-71 (1992)

24. Zagatti, S.: The Cauchy problem for Hartree-Fock time-dependent equations. Ann. Inst. Henri Poincaré (A) 56, 357-374 (1992) 